\title{
Global Innovation Monitor
}

des Rates für Forschung und

Technologieentwicklung

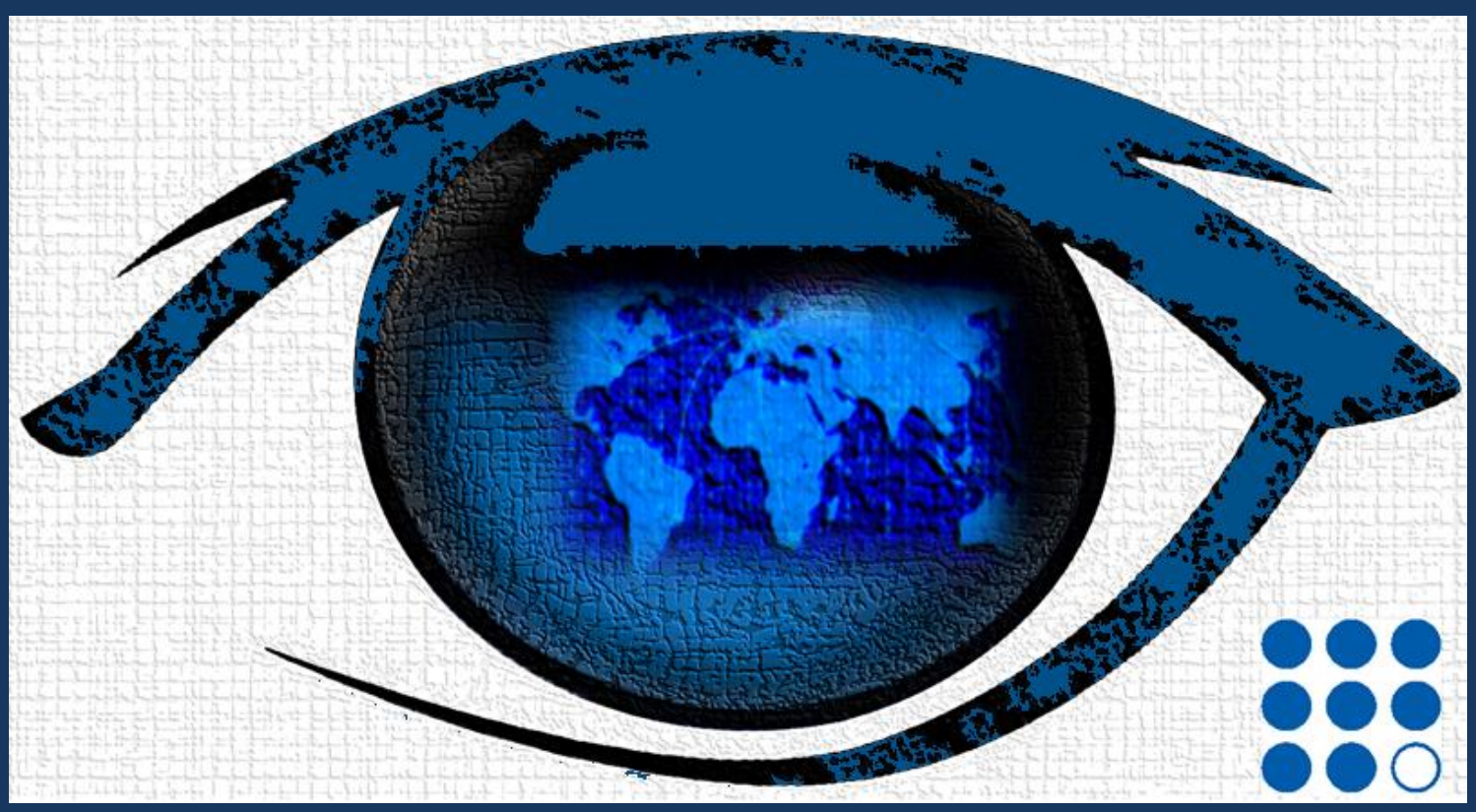


IMPRESSUM

HERAUSGEBER UND MEDIENINHABER: RAT FÜR FORSCHUNG UND TECHNOLOGIEENTWICKLUNG,

PESTALOZZIGASSE 4, 1010 WIEN

INHALTLICHE BERATUNG UND PROZESSBEGLEITUNG: ÖSTERREICHISCHES INSTITUT FÜR WIRTSCHAFTSFORSCHUNG

WIEN, AUGUST 2014 


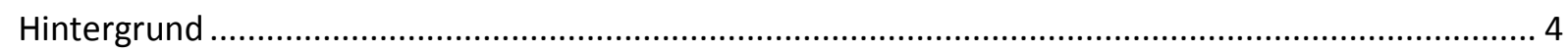

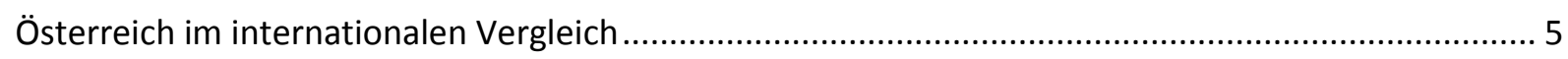

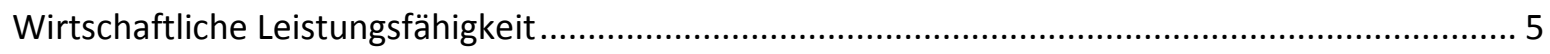

Wissenschaftliche und technologische Leistungsfähigkeit ...................................................... 6

Gesellschaftliches Wohlergehen, Lebensqualität und Lebenserwartung......................................... 8

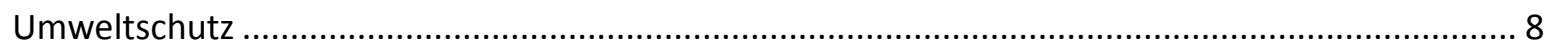

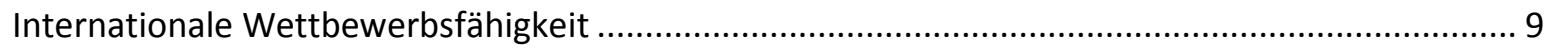

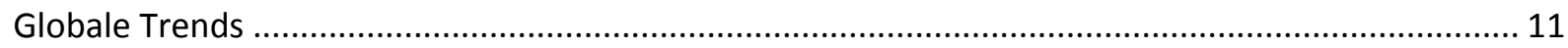

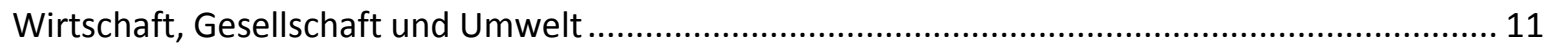

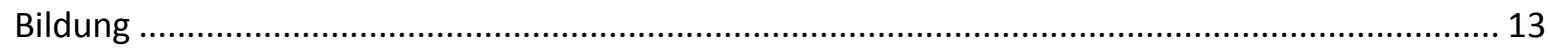

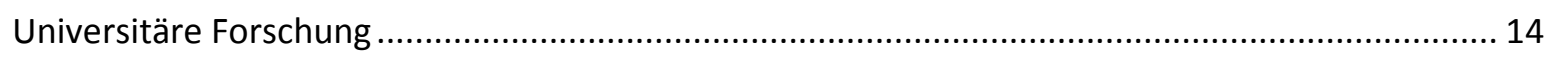

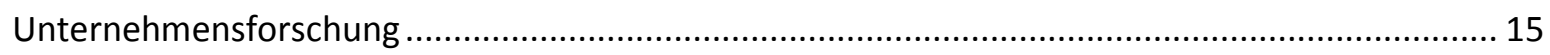

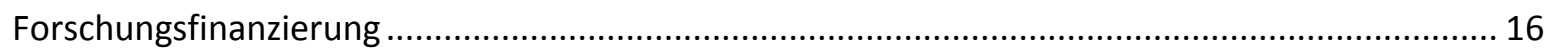

Trends in Österreich im Vergleich mit ausgewählten Ländern ...................................................... 18

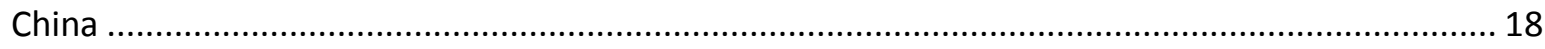

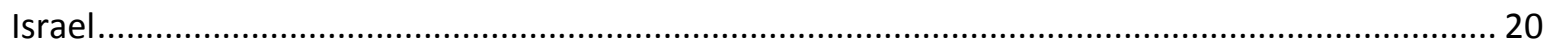

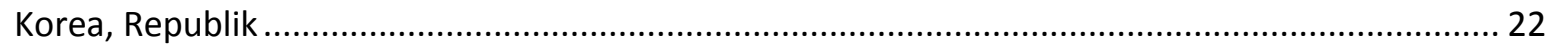

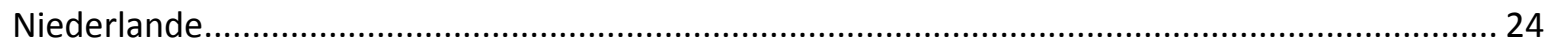

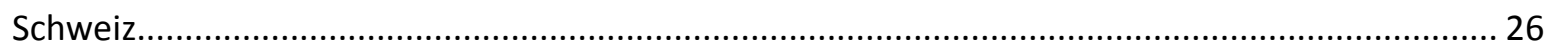

USA

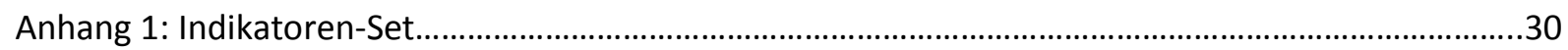

Anhang 2: Erläuterungen zu Methodik und Interpretation der Abbildungen und Indikatoren.............31 
Die Innovationsfähigkeit eines Landes hängt von mehreren, sich ergänzenden Faktoren ab, deren Zusammenspiel erst Erfolg oder Misserfolg im internationalen Wettbewerb bewirken. Als wichtige Kriterien gelten dabei ein flexibles und motivierendes Bildungssystem, qualifizierte Arbeitskräfte, eine enge Zusammenarbeit von Wissenschaft und Wirtschaft sowie eine flexible politische Steuerung, die optimale Rahmenbedingungen ermöglicht.

Die Positionierung Österreichs im internationalen Vergleich wird dabei in einer globalisierten Wissenschafts- und Wirtschaftswelt von immer größerer Bedeutung. Wachstum, Beschäftigung und Wohlstand sind gleichermaßen von Produkt- und Prozessinnovationen abhängig, werden von sozialen Innovationen unterstützt und erfordern einen hohen Forschungs- und Entwicklungsaufwand.

Das Vorhaben der Bundesregierung, Österreich zu einer der leistungsfähigsten Forschungs- und Technologienationen zu machen, bleibt laut Regierungsübereinkommen eines der wichtigsten Ziele. Die im März 2011 präsentierte FTI-Strategie „Der Weg zum Innovation Leader" bildet dafür die Grundlage und bleibt daher weiterhin richtungsweisend. Der Rat für Forschung und Technologieentwicklung führt im Auftrag des Ministerrats ein jährliches Monitoring der wissenschaftlichen und technologischen Leistungsfähigkeit Österreichs im internationalen Vergleich durch. ${ }^{1}$ Gleichzeitig beobachtet der Rat die Fortschritte bei der Umsetzung der FTI-Strategie und bewertet die getroffenen Maßnahmen sowie deren Zielorientierung. Dabei bilden die europäischen Innovation Leaders Dänemark, Deutschland, Finnland und Schweden den Benchmark.

Ein Vergleich Österreichs mit den führenden Innovationsnationen nicht nur auf europäischer Ebene, sondern auch im globalen Kontext soll dazu beitragen, den Status Österreichs im Lichte weltweiter Trends zu beleuchten. Das ist das eigentliche Ziel des "Global Innovation Monitors", der hier erstmals präsentiert wird und ab 2015 fixer Bestandteil des „Berichts zur wissenschaftlichen und technologischen Leistungsfähigkeit Österreichs" werden soll.

Als erstes Set für einen globalen Vergleich wurden die Länder China, Israel, Niederlande, Schweiz, Südkorea und die USA ausgewählt - und das aus mehreren Gründen: Erstens wurden sie als Zielländer der Prioritäten 1 und 2 aus den Empfehlungen der AG 7a an die Task Force FTI definiert. ${ }^{2}$ Zweitens gelten die ausgewählten Länder - bis auf China - als globale Innovation Leaders, die aufgrund ihrer erfolgreichen Performance als Benchmark dienen können. China wurde deswegen ausgewählt, weil es wie kein zweites Land rasante Wachstumsraten in Hinblick auf Forschungsausgaben, Publikationen, Patente etc. aufweist. Drittens war der Rat in diesen Ländern an Delegationsreisen beteiligt, die tiefere Einblicke in die dortigen FTI-Systeme gewährt haben. Und schließlich liegen für alle ausgewählten Nationen Kennzahlen vor, die im Rahmen von Datenbeständen der OECD etc. regelmäßig erhoben, aktualisiert und öffentlich zugänglich gemacht werden (siehe dazu das Indikatoren-Set in Anhang 1).

${ }^{1}$ Bericht zur wissenschaftlichen und technologischen Leistungsfähigkeit Österreichs 2014, 2013, 2012

2 Beyond Europe: Die Internationalisierung Österreichs in Forschung, Technologie und Innovation über Europa hinaus. Empfehlungen der AG 7a an die Task Force der Bundesregierung (Juli 2013), S. 7. 


\section{WIRTSCHAFTLICHE LEISTUNGSFÄHIGKEIT}

Österreich ist - gemessen an den gängigen ökonomischen Kennzahlen - eines der erfolgreichsten Länder der Welt. Österreichs Wirtschaftswachstum liegt seit über zehn Jahren kontinuierlich über dem Durchschnitt der Eurozone. Seit dem Jahr 2000 wuchs die österreichische Wirtschaft im Schnitt real um 1,5\% pro Jahr. Das liegt zwar um gut einen Prozentpunkt unter den Wachstumsraten der beiden Dekaden davor, ${ }^{3}$ aber deutlich über jenen Westeuropas und Deutschlands. Im Vergleich dazu ist die europäische Wirtschaft lediglich um rund 1\%, die deutsche sogar nur um 0,9\% gewachsen. Über die letzten zehn Jahre kumuliert ergibt das einen Wachstumsvorsprung Österreichs von fünf bzw. sieben Prozentpunkten. Das österreichische Wachstum kam damit fast an das US-amerikanische heran.

Im Ländervergleich weist Österreich eine hohe Beschäftigungsquote, eine überdurchschnittlich niedrige Arbeitslosigkeit und substanziellen Wohlstand (gemessen an einem überdurchschnittlich hohen BIP pro Kopf) ${ }^{4}$ auf, womit es in diesen Bereichen weiterhin eine führende Position in Europa und der Welt innehält. Dieses Wachstum geht einher mit einer im internationalen Vergleich relativ geringen Einkommensungleichheit (gemessen am Gini-Koeffizient), substanziellen Umwelt-Standards und einer hohen Lebenserwartung.

Dieser wirtschaftliche Erfolg darf allerdings nicht darüber hinwegtäuschen, dass sich Österreich nun in einer Stagnationsphase befindet. Die bisherige Entwicklungsdynamik ist seit 2009 verloren gegangen. Bemerkbar macht sich dies etwa bei der Entwicklung der Lohnstückkosten: Diese verlief bis 2005 im Gleichklang mit Deutschland, entwickelt sich aber seither deutlich schlechter. In Österreich stiegen die Lohnstückkosten zwischen 2005 und 2012 um rund 14\%, verglichen mit rund $6 \%$ in Deutschland, rund $8 \%$ in der Schweiz und 10\% in Schweden. Weiters hat Österreich mit 5,4\% des BIP eine doppelt so hohe Subventionsquote wie der EU-Schnitt.

Außerdem weist Österreich mit rund $43 \%$ - verglichen mit rund $39 \%$ im EU-Durchschnitt oder rund 38\% der Schweiz - eine der höchsten Abgabenquoten im Ländervergleich auf. Hier ist vor allem die hohe Abgabenbelastung des Faktors Arbeit kritisch, wodurch die Beschäftigung von schlecht ausgebildeten Personen (und damit der unteren Einkommen) gehemmt wird. Der IWF kritisiert in

\footnotetext{
${ }^{3}$ Dies ist nicht zuletzt darauf zurückzuführen, dass die konjunkturellen Zyklen (Auf-/Abschwung) in fast allen industrialisierten Ländern seit Beginn der neunziger Jahre eine geringere Amplitude aufweisen.

${ }^{4}$ Gemessen am Pro-Kopf-Einkommen liegt Österreich in der EU an dritter Stelle und weltweit auf dem 11. Platz.
} 
seinem neuesten Länderbericht einen Rückgang der Produktivität seit Mitte der Neunziger-Jahre, ${ }^{5}$ die mittlerweile rund $20 \%$ unter jener der USA liegt. ${ }^{6}$

Aufgrund seiner überdurchschnittlich hohen Ausgabenquote ist der öffentliche Sektor in großem Ausmaß für die hohe Staatsverschuldung verantwortlich. Der EU-Stabilitätspakt sieht eine Verschuldungsgrenze von höchstens 60 Prozent des Bruttoinlandsprodukts vor. Österreich liegt laut Statistik Austria mit offiziell 74,5\% (Stand 2013) jedoch deutlich darüber. Tatsächlich ist die Quote in Folge der Ausgliederungen von ÖBB, ASFINAG, BIG etc. sowie durch zusätzliche Kosten des Familienlastenausgleichs oder der Bankenrettung deutlich höher; gleichzeitig darf man auch die privaten Schulden nicht außer Acht lassen.

\section{WISSENSCHAFTLICHE UND TECHNOLOGISCHE LEISTUNGSFÄHIGKEIT}

Österreichs wissenschaftliche und technologische Leistungsfähigkeit hat sich seit den 1980er Jahren kontinuierlich gesteigert. Eine Vielzahl an Analysen $^{7}$ bescheinigt dem österreichischen Innovationssystem eine stetig verbesserte Performance, die sich auch in einem gesteigerten Output niederschlägt. Betrachtet man etwa die Anzahl an Publikationen in Relation zu Bevölkerungszahl und wissenschaftlichem Impact oder beispielsweise die Anzahl wissens- und technologiebasierter Exporte, so zählt Österreich zu den führenden Ländern der Welt. Der Beitrag von medium- und hightech-Produkten zur Handelsbilanz ist in Österreich überdurchschnittlich hoch und liegt in etwa auf gleicher Höhe mit den Werten Deutschlands oder der Schweiz. Dank dieser beachtlichen Exportleistungen, vor allem aus dem industriellen Bereich, mit einem Volumen von rund 125,4 Milliarden Euro - wozu noch industrienahe Dienstleistungsexporte im Ausmaß von etwa 50 Milliarden dazukommen - erfreut sich die österreichische Volkswirtschaft schon seit längerem eines deutlichen Leistungsbilanzüberschusses. Eine Folge davon ist unter anderem die Rekordzahl an Erwerbstätigen.

Aus den diversen Rankings zur Messung der Innovationsperformance wird jedoch auch deutlich, dass die Innovationsanstrengungen nicht ausreichen, um in die Gruppe der führenden Länder vorzustoßen. Dies verdeutlicht etwa der Innovation Union Scoreboard (IUS): Österreich ist im Vergleich zum Vorjahr neuerlich um einen Platz zurückgefallen und liegt nun auf Rang 10. Das ist der vierte Rückfall in Folge. Grund dafür ist die Tatsache, dass Belgien, die Niederlande, Irland und UK in den letzten Jahren stärker als Österreich gewachsen sind und Österreich überholt haben. Die vier

\footnotetext{
${ }^{5}$ International Monetary Fund, Austria Article IV Consultation Preliminary Conclusions.

6 Vgl. Bank of International Settlements (2014): Yearly Report 2014, 64ff. Zwar schneidet Österreich beim Beschäftigungswachstum in den letzten Jahren gut ab, da aber die Wirtschaftsleistung verhältnismäßig schwächer zulegte, schrumpfte die Produktivität gemessen am Output pro Beschäftigtem um 4.2\%. Zum Vergleich: In Deutschland hielten sich Beschäftigungszuwachs und Wirtschaftswachstum die Waage, in Frankreich verbesserte sich die Produktivität um gut zwei Prozent. Die BIZ empfiehlt dringend die Implementierung struktureller Maßnahmen zur Erhöhung der Produktivität.

${ }^{7}$ Bericht zur wissenschaftlichen und technologischen Leistungsfähigkeit Österreichs 2014, S. 72f.; vgl. auch Forschungs- und Technologiebericht 2014, 2013, 2012 etc.; WIFO, prognos, convelop, KMU Forschung Austria (2009): Systemevaluierung des österreichischen Innovationssystems.
} 
Innovation Leaders Schweden, Dänemark, Deutschland und Finnland bleiben weiterhin in Führung. Zwar verringert sich der relative Rückstand zu den führenden Ländern, weil Österreich bezogen auf den Gesamtindex seit 2006 schneller als die Innovation Leaders gewachsen ist. Diese liegen jedoch im Level noch weit vorne.

Das Innovationsmonitoring des Rates kommt daher zu dem Schluss, dass die österreichische Aufholdynamik derzeit unzureichend ist. Will Österreich im globalen Wettbewerb nicht weiter zurückfallen und den Anschluss an die Spitzengruppe nicht verlieren, muss den Themen Bildung, Forschung, Technologie und Innovation höchste Priorität eingeräumt, die dafür erforderliche Finanzierung bereitgestellt und auch Strukturanpassungen vorgenommen werden. Aus der Analyse des Rates lassen sich vier prioritäre Handlungsfelder ableiten: Die Performance des Bildungssystems muss verbessert werden, der tertiäre Bildungssektor muss deutlich stärker ausgestattet werden, die Mittel für die kompetitive Finanzierung der Grundlagenforschung - und parallel dazu auch für die angewandte Forschung - müssen angehoben werden, die Gründungsdynamik muss gesteigert und der Anteil der privaten Finanzierung von F\&E muss erhöht werden. 
Österreich ist ein Land mit überdurchschnittlich hohem gesellschaftlichem Wohlergehen, hoher Lebensqualität und hoher Lebenserwartung. Dies wird etwa durch den Better Life Index der OECD zum Ausdruck gebracht, ${ }^{8}$ demzufolge sich Österreich unter den 15 Ländern mit dem besten gesellschaftlichen Wohlergehen und der höchsten Lebensqualität befindet. Allerdings befinden sich die Schweiz, die Niederlande, die USA und Deutschland klar vor Österreich.

Der Human Development Index (HDI) der Vereinten Nationen weist Österreich mit Rang 21 ebenfalls eine Position unter den Top-Nationen der 187 erfassten Ländern der Welt aus. ${ }^{9}$ Es befindet sich damit in der 49 Länder umfassenden Kategorie der "sehr hoch entwickelten Staaten“. Österreich schneidet in den meisten Kategorien überdurchschnittlich gut ab. Allerdings rangieren alle für den „Global Innovation Monitor" ausgewählten Länder mit Ausnahme Chinas vor Österreich. Zum Vergleich: Die Schweiz liegt auf Platz 3, die Niederlande auf Platz 4, die USA auf Platz 5, Südkorea auf Platz 16 und Israel auf Platz 19.

Im Bereich der Lebenserwartung zählt Österreich ebenso zu einem der weltweit führenden Länder. Laut World Population Prospects der UNO ${ }^{10}$ liegt die Lebenserwartung in Österreich bei etwas über 80 Jahren. Hier zeigt sich in den letzten Jahren eine klare Verbesserung vor allem für Frauen, in geringerem Ausmaß aber auch für Männer. Die Entwicklungsdynamik für Frauen ist allerdings deutlich ausgeprägter, weshalb deren Anteil an Lebensjahren ohne Beeinträchtigung durch Krankheit bereits gleichauf mit jenem der führenden Länder wie der Schweiz, Schweden, Israel oder den Niederlanden liegt.

\section{UMWELTSCHUTZ}

Die Bilanz des OECD-Umweltprüfberichts für Österreich ${ }^{11}$ fällt in den meisten Bereichen positiv aus: die Trinkwasserqualität gehört zu den besten weltweit, der Anteil erneuerbarer Energien am Gesamtenergieaufkommen ist dreimal so hoch wie im OECD-Durchschnitt und der Ressourcenverbrauch ist gemessen am Bruttoinlandsprodukt moderat. Mit einem Gesamtumsatz von 33 Mrd. Euro und 170.000 "green jobs" hat die Öko-Wirtschaft den Tourismus an Bedeutung überholt. Die Umweltindikatoren zeigen eine leichte Verbesserung der Ressourcenproduktivität und eine Stagnation bei Energieintensität und Treibhausgasemissionen. Bei der Ressourcenproduktivität hat sich der Zielabstand zu den führenden Ländern verringert, die Dynamik ist jedoch derzeit nicht

\footnotetext{
${ }^{8}$ Der Better Life Index umfasst Indikatoren aus elf Teilbereichen wie Bildung, Umwelt, Gesundheit, Lebenszufriedenheit, Sicherheit, Work-Life-Balance etc. Vgl. http://www.oecdbetterlifeindex.org/de/

${ }^{9}$ Der Human Development Index misst den Entwicklungsstand eines Landes anhand alternativer Werte wie Bildung, Ernährung, Gesundheit oder Möglichkeiten der Mitbestimmung. Vgl. http://hdr.undp.org/en/data

10 http://esa.un.org/wpp/index.htm

${ }^{11}$ OECD (2013): Environmental Performance Review Austria.
} 
ausreichend, um die Innovation Leaders bis 2020 einzuholen. Im Bereich der Energieeffizienz hat Österreich etwas an Boden verloren. Aufgrund der weiterhin ungünstigen Wachstumsdynamik sind nach wie vor zusätzliche Maßnahmen für die Senkung der Treibhausgase notwendig. Hier sind die führenden Innovationsnationen in den vergangenen Jahren konsequent in Richtung der Kyoto-Ziele allen voran die Reduktion der Emission von Treibhausgasen auf 13\% unter den Wert von 1990 unterwegs.

Um zu den Spitzenländern aufzuschließen, fordert die OECD Österreich dazu auf, eine sozialökologische Steuerreform zu implementieren, die den Faktor Arbeit entlasten und umweltschädliches Verhalten/public bads (Stichwort: Pendlerpauschale) stärker belasten sollte. Zu den wichtigsten Herausforderungen im Umweltsektor gehört für die OECD die Reduktion der Treibhausgasemissionen. Ein Drittel davon wird durch den motorisierten Individualverkehr verursacht. Knapp ein Drittel der verkehrsbedingten Emissionen wiederum gehen auf den TankTourismus zurück, der primär durch die im Vergleich zu den Nachbarländern niedrigen Kraftstoffpreise bzw. -steuern in Österreich verursacht wird.

\section{INTERNATIONALE WETTBEWERBSFÄHIGKEIT}

Die österreichische Wirtschaftskammer hat in ihrem "Monitoring Report 2014“12 über 120 internationale Rankings zur Wettbewerbsfähigkeit der österreichischen Wirtschaft und Gesellschaft zusammengestellt. Fazit der Analyse: Seit 2005 sinkt die österreichische Wettbewerbsfähigkeit im globalen Vergleich. Lag man 2005 noch unter den Top 20\%, so befindet sich Österreich 2014 nur mehr knapp im vorderen Drittel aller Länder. Dies lässt sich exemplarisch an der Entwicklung der Positionierung Österreichs im „World Competitiveness Report 2014“"13 verdeutlichen: Die beste Platzierung erzielte Österreich im Jahr 2007 mit Platz 11, ab dann ging es kontinuierlich bergab. Zwar hat sich die Platzierung gegenüber dem Vorjahr um einen Platz leicht verbessert, trotzdem liegt Österreich heute nur mehr auf Rang 22 (siehe Abbildung 1).

Die größten Verbesserungen im Vergleich zum Vorjahr lieferten eindeutig die Unternehmen. Während die Leistungsfähigkeit der heimischen Volkswirtschaft durchwegs besser benotet wurde und gleich um fünf Plätze auf Rang 17 kletterte, stagnierte die Bewertung der Kompetenz der Regierung und der Qualität der Corporate Governance auf Platz 36. Besonders schlecht bewertet wird die heimische Budgetpolitik. Hier landet Österreich nur auf Platz 58 unter den 60 untersuchten Ländern. Die größten Hemmnisse sind demnach die reale Steuerbelastung, die Alterung der Bevölkerung und die Finanzierung der Pensionen. Die effektive Steuerbelastung für Arbeitseinkommen wird, analog zu Befunden der OECD und des IWF, überhaupt in keinem anderen Land schlechter bewertet. Am besten bewertet wird die Produktivität und Effizienz der Unternehmen. Hier landet Österreich auf dem 9. Platz. Beim internationalen Handel kletterte

\footnotetext{
12 WKÖ (2014): Monitoring Report 2014

13 IMD (2014): World Competitiveness Report.
} 
Österreich gar vom 22. auf den zehnten Platz nach oben. Auch die Kapitalflüsse aus dem Ausland, die stärkere Kapitalisierung der Wiener Börse, die gut ausgebildeten Fachkräfte im Land, das hohe Bildungsniveau, ein effektives Rechtsumfeld und gute Arbeitsbedingungen werden von den Studienautoren positiv beurteilt.

Die größten Herausforderungen für Österreich sieht das IMD im Bereich der Politik: Das tatsächliche Pensionsantrittsalter müsse steigen, Energiekosten müssten sinken, das Steuersystem, die Verwaltungsstrukturen und das Schulsystem reformiert sowie Engpässe in der höheren Schulbildung endlich gelöst werden.

Von den sechs ausgewählten Ländern China, Israel, Niederlande, Schweiz, Südkorea und USA liegen China, Israel und Korea unmittelbar hinter Österreich auf den Plätzen 23, 24 und 26. Die übrigen zählen zu den Top 15. Die Schweiz konnte sich erneut auf Platz 2 behaupten, nur knapp geschlagen von den USA, die im IMD-Ranking ihre Spitzenposition als wettbewerbsstärkstes Land verteidigten. Deutschland, das noch vor wenigen Jahren als „kranker Mann Europas" bezeichnet wurde, stieß weiter vom 9. auf den 6. Platz vor und liegt nur marginal hinter den fünftplatzierten Schweden zurück. Die Niederlande liegen weiter auf Platz 14.

Abbildung 1: IMD-Ranking 2007-2014

\begin{tabular}{|c|c|c|c|c|c|c|c|c|}
\hline & 2007 & 2008 & 2009 & 2010 & 2011 & 2012 & 2013 & 2014 \\
\hline 1 & USA & USA & USA & Singapore & USA & Hong Kong & USA & USA \\
\hline 2 & Singapore & Singapore & Hong Kong & Hong Kong & Hong Kong & USA & Switzerland & Switzerland \\
\hline 3 & Hong Kong & Hong Kong & Singapore & USA & Qatar & Switzerland & Hong Kong & Singapore \\
\hline 4 & Luxembou & Switzerland & Switzerland & Switzerland & Singapore & Singapore & Sweden & Hong Kong \\
\hline 5 & Denmark & Luxembourg & Denmark & Australia & Sweden & Sweden & Singapore & Sweden \\
\hline 6 & Switzerlan & Denmark & Sweden & Qatar & Switzerland & Canada & Norway & Germany \\
\hline 7 & Iceland & Australia & Australia & Sweden & Taiwan & Taiwan & Canada & Canada \\
\hline 8 & Netherlan & Canada & Canada & Canada & Canada & Norway & UAE & UAE \\
\hline 9 & Sweden & Sweden & Qatar & Taiwan & Australia & Germany & Germany & Denmark \\
\hline 10 & Canada & Netherlands & Finland & Norway & Germany & Qatar & Qatar & Norway \\
\hline 11 & Austria & Norway & Netherlands & Malaysia & Luxembourg & Netherlands & Taiwan & Luxembourg \\
\hline 12 & Australia & Ireland & Norway & Iceland & Denmark & Luxembourg & Denmark & Malaysia \\
\hline 13 & Norway & Taiwan & Luxembourg & Luxembourg & Norway & Denmark & Luxembourg & Taiwan \\
\hline 14 & Ireland & Austria & Germany & Netherlands & Netherlands & Malaysia & Netherlands & Netherlands \\
\hline 15 & Qatar & Finland & NZL & Denmark & Peru & Australia & Malaysia & Ireland \\
\hline 16 & China & Qatar & Austria & Austria & Finland & UAE & Australia & UK \\
\hline 17 & Germany & Germany & Japan & Germany & Malaysia & Finland & Ireland & Australia \\
\hline 18 & Finland & China & Malaysia & Israel & Israel & UK & UK & Finland \\
\hline 19 & Taiwan & NZL & Ireland & China & Austria & Israel & Israel & Qatar \\
\hline 20 & NZL & Malaysia & China & Finland & China & Ireland & Finland & NZL \\
\hline 21 & UK & Israel & UK & NZL & UK & Austria & China & Japan \\
\hline 22 & Israel & UAE & Belgium & Ireland & NZL & Korea & Korea & Austria \\
\hline 23 & Estonia & UK & Taiwan & UK & Korea & China & Austria & China \\
\hline 24 & Malaysia & Japan & Israel & Korea & Belgium & NZL & Japan & Israel \\
\hline 25 & Japan & Estonia & Chile & France & Ireland & Belgium & NZL & Iceland \\
\hline
\end{tabular}

Quelle: International Institute for Management Development (IMD) 


\section{GLOBALE TRENDS}

Die global innovativsten Länder, allen voran die USA, Deutschland, die skandinavischen Staaten, die Schweiz und Südkorea haben bereits seit längerem erkannt, dass der Weg zur Bewältigung der globalen Grand Challenges nur über verstärkte Anstrengungen in den Bereichen Bildung, Forschung und Innovation verläuft. Daher haben diese Länder auch und gerade in der Zeit der Wirtschaftskrise ihre Investitionen in Forschung und Entwicklung (F\&E) und Bildung massiv erhöht. Diese Investitionen kommen auch der wirtschaftlichen Leistungsfähigkeit und dem gesellschaftlichen Wohlergehen zugute. ${ }^{14}$

Für den „Global Innovation Monitor“ werden in Anlehnung an den „Bericht zur wissenschaftlichen und technologischen Leistungsfähigkeit Österreichs“ Indikatoren aus den Bereichen Wirtschaft, Gesellschaft, Umwelt, Bildung, universitäre Forschung und Unternehmensforschung für ausgewählte Länder (China, Israel, Niederlande, Schweiz, Südkorea, USA) hinsichtlich ihrer Entwicklung mit Österreich verglichen. Im Folgenden wird die Leistungsfähigkeit der genannten Bereiche dieser Länder in Relation zu Österreich abgebildet.

\section{WIRTSCHAFT, GESELLSCHAFT UND UMWELT}

Abbildung 2 gibt einen Überblick über die Entwicklungstrends der im „Global Innovation Monitor“ näher behandelten Länder in Bezug auf ihre Leistungsfähigkeit in den Bereichen Wirtschaft, Gesellschaft und Umwelt. Die Berechnung des Zielabstandes zu AT (x-Achse) bzw. der Entwicklungsdynamik ( $y$-Achse) erfolgt normiert zu Österreich (=100). Jene Länder, die aktuell besser als Österreich bewertet werden, liegen in dieser Darstellung rechts von 100. Mit einem y-Achsenwert $>100$ weisen die gezeigten Länder eine höhere Entwicklungsdynamik als Österreich auf und verringern dadurch ihren Abstand zu Österreich bzw. können nicht eingeholt werden.

14 Vgl. IHS (2013): Vision Österreich 2050: Vorsprung durch Bildung, Innovation und Wandel; Androsch, H. / Gadner, J. (2013): Die Zukunft Österreichs in der Welt von Morgen. In: Rat für Forschung und Technologieentwicklung (Hg.): Österreich 2050 - FIT für die Zukunft. Holzhausen, Wien. S. 254-272, $259 f$. 


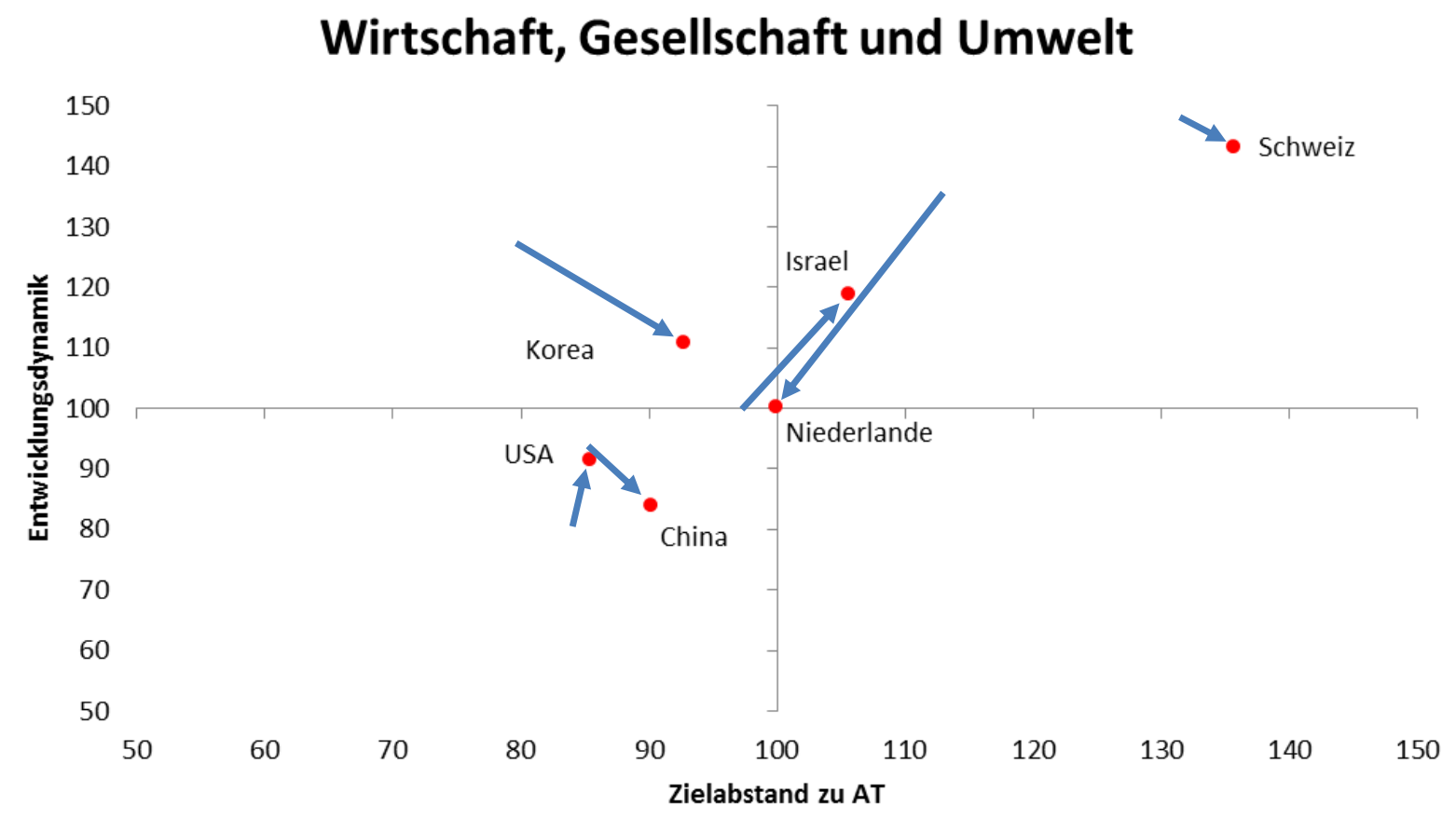

Quelle: siehe Indikatorenliste Anhang 1, WIFO-Darstellung; Erläuterungen zu Methodik und Interpretation der Abbildungen und Indikatoren siehe Anhang 2

Aus der Abbildung wird ersichtlich, dass Österreich unter Berücksichtigung der wirtschaftlichen, gesellschaftlichen und umweltrelevanten Kennzahlen verhältnismäßig gut positioniert ist. Lediglich die Schweiz und Israel stehen heute in Bezug auf ihre wirtschaftliche Entwicklung, ihr gesellschaftliches Wohlergehen und die Umweltsituation besser da, die Niederlande liegen in etwa gleichauf mit Österreich. Die Entwicklungsdynamik Koreas ist allerdings höher als jene Österreichs, weshalb Korea bei fortlaufendem Trend Österreich bis 2020 einholen könnte. Die Performance der USA ist trotz der Steigerungen nicht ausreichend, um Österreich bis 2020 überholen zu können. Der Entwicklungstrend Chinas ist bezogen auf die Chance, Österreich bis ins Jahr 2020 zu überholen, sogar rückläufig. Allein aufgrund dieses Rückstands zu Österreich sollte jedoch die Wachstumsdynamik Chinas nicht unterschätzt werden. 


\section{BILDUNG}

Die Bewertung des Bildungssystems stellt in aggregierter Form die Ergebnisse der PISAUntersuchungen als Outputkomponente und die Ausgaben im tertiären Bildungssektor für Studierende als Inputkomponente dar. Vergleicht man die aktuellen Bewertungen (Werte 2012), liegt Österreichs Bildungsperformance vor Israel, Korea und China, jedoch hinter den USA, den Niederlanden und der Schweiz.

Abbildung 3: Bildung im internationalen Vergleich

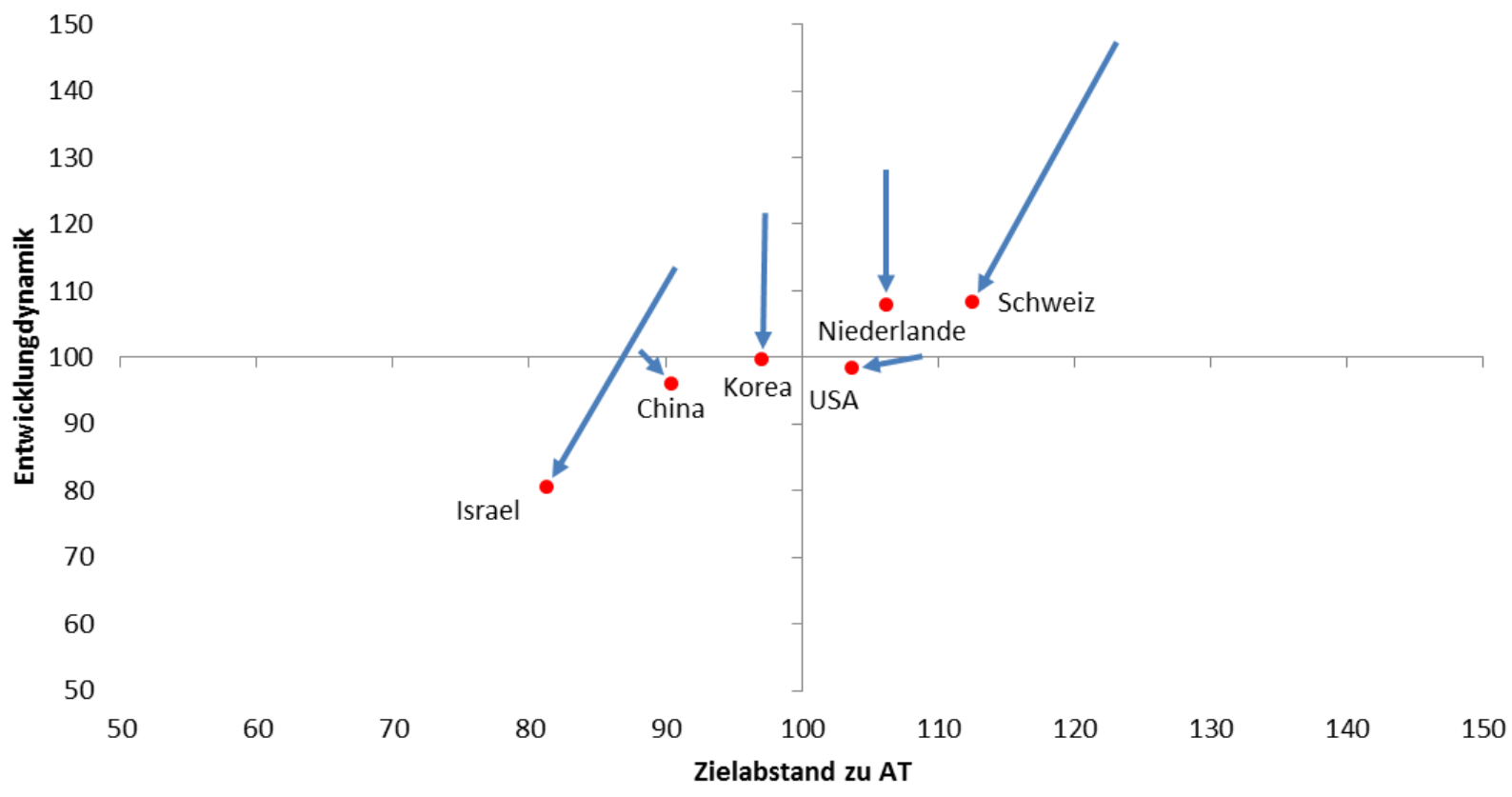

Quelle: siehe Indikatorenliste Anhang 1, WIFO-Darstellung; Erläuterungen zu Methodik und Interpretation der Abbildungen und Indikatoren siehe Anhang 2

Obwohl die Leistungsfähigkeit des österreichischen Bildungssystems in diesen Indikatoren in Relation zu allen betrachteten Ländern gestiegen ist, bleiben die Niederlande und die Schweiz in ihrer Entwicklungsdynamik klar vor Österreich. Korea, China und die USA liegen etwa gleich auf. Die Abbildung zeigt aber auch, dass die Dynamik aller betrachteten Länder über den gesamten Zeitraum hoch war. Eine kontinuierliche Steigerung der Qualität in der Ausbildung und ein verstärkter Mitteleinsatz insbesondere im tertiären Bildungsbereich bleiben daher ein essentieller Faktor für die Bildungspolitik in Österreich. 


\section{UNIVERSITÄRE FORSCHUNG}

Der Trend im Bereich der universitären Forschung stellt sich ähnlich dar wie jener im Bereich der Bildung. Auf Basis der aggregierten Einzelindikatoren „Hochschulranking“, „Ausgaben für Grundlagenforschung" und „Ausgaben für tertiäre Bildung, Forschung und Entwicklung in Relation zur Bevölkerung" liegt Österreich hinter der Schweiz, Israel, den Niederlanden und den USA. Bis zum Jahr 2020 könnte Österreich aber Israel, die USA und die Niederlande einholen.

China und Korea liegen hinter Österreich. Insbesondere Korea zeichnet sich durch enorm hohe Steigerungsraten aus, was dazu führen wird, dass Korea bis zum Jahr 2020 Österreich eingeholt haben wird. Die Dynamik für die Niederlande, die USA und Israel sind in der Bewertung für 2012 unter der Österreichs, allerdings auf hohem Niveau. Chinas Abstand erklärt sich durch die hohe Bevölkerungszahl und die sehr heterogenen Entwicklungen in diesem Land. Der absolute Mitteleinsatz für universitäre Forschung und tertiäre Ausbildung ist in China allerdings enorm und führt global zu einer markanten Verschiebung in der Verfügbarkeit von hoch ausgebildeten ForscherInnen.

Abbildung 4: Universitäre Forschung im internationalen Vergleich

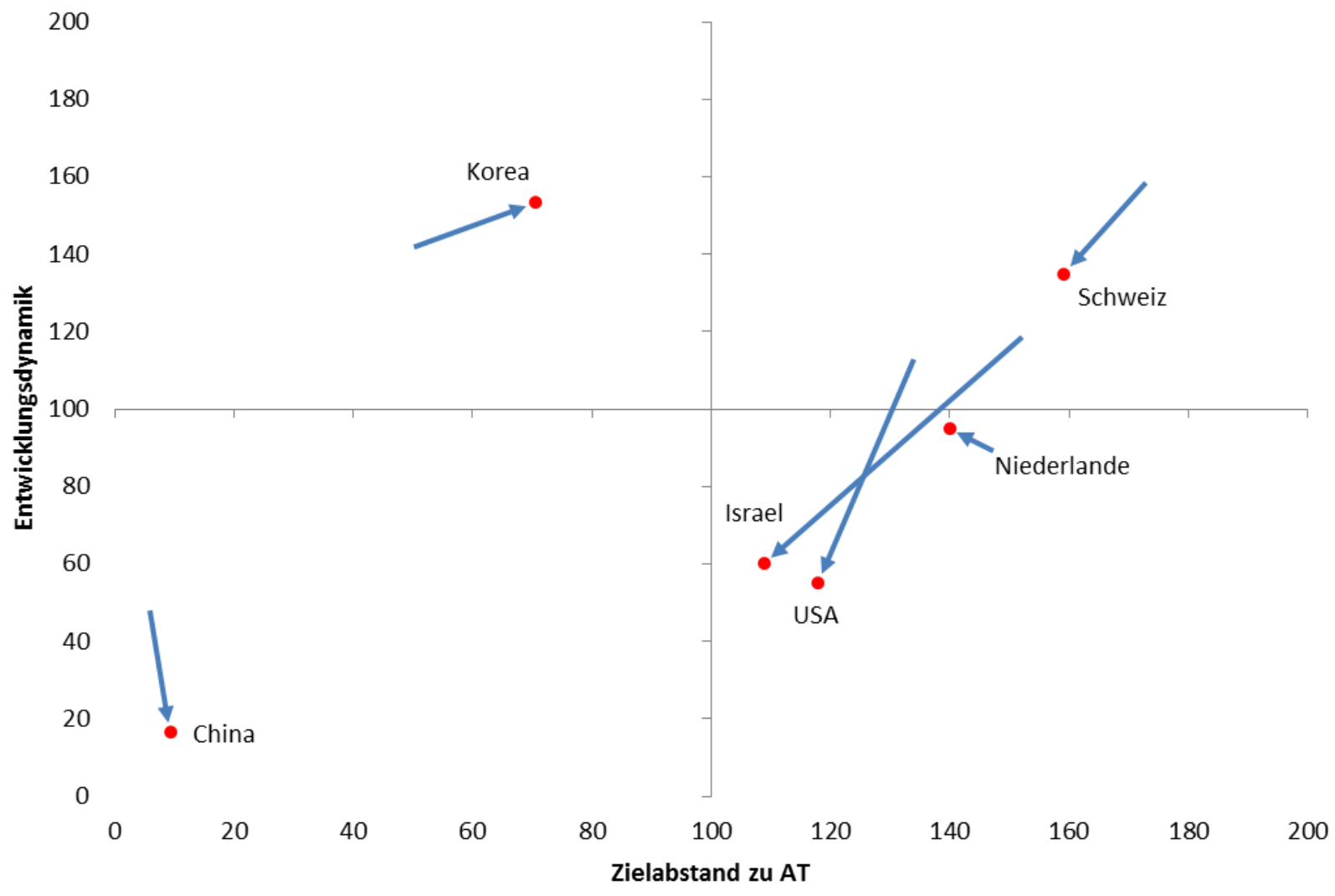

Quelle: siehe Indikatorenliste Anhang 1, WIFO-Darstellung; Erläuterungen zu Methodik und Interpretation der Abbildungen und Indikatoren siehe Anhang 2 


\section{UNTERNEHMENSFORSCHUNG}

Abbildung 5 zeigt für Österreich einen Aufholbedarf hinsichtlich der Performance in den Indikatoren für Unternehmensforschung und Innovation, die für diese Darstellung zusammengefasst wurden. Bis auf China liegen alle ausgewählten Länder vor Österreich und vier davon - Israel, Korea, USA und die Niederlande - werden aufgrund ihrer dynamischen Entwicklung ihren Vorsprung auf Österreich weiter ausbauen. Bei einem gleichbleibenden Trend im Bereich der Unternehmensforschung kann auch China weiter zu Österreich aufschließen.

Abbildung 5: Unternehmensforschung im internationalen Vergleich

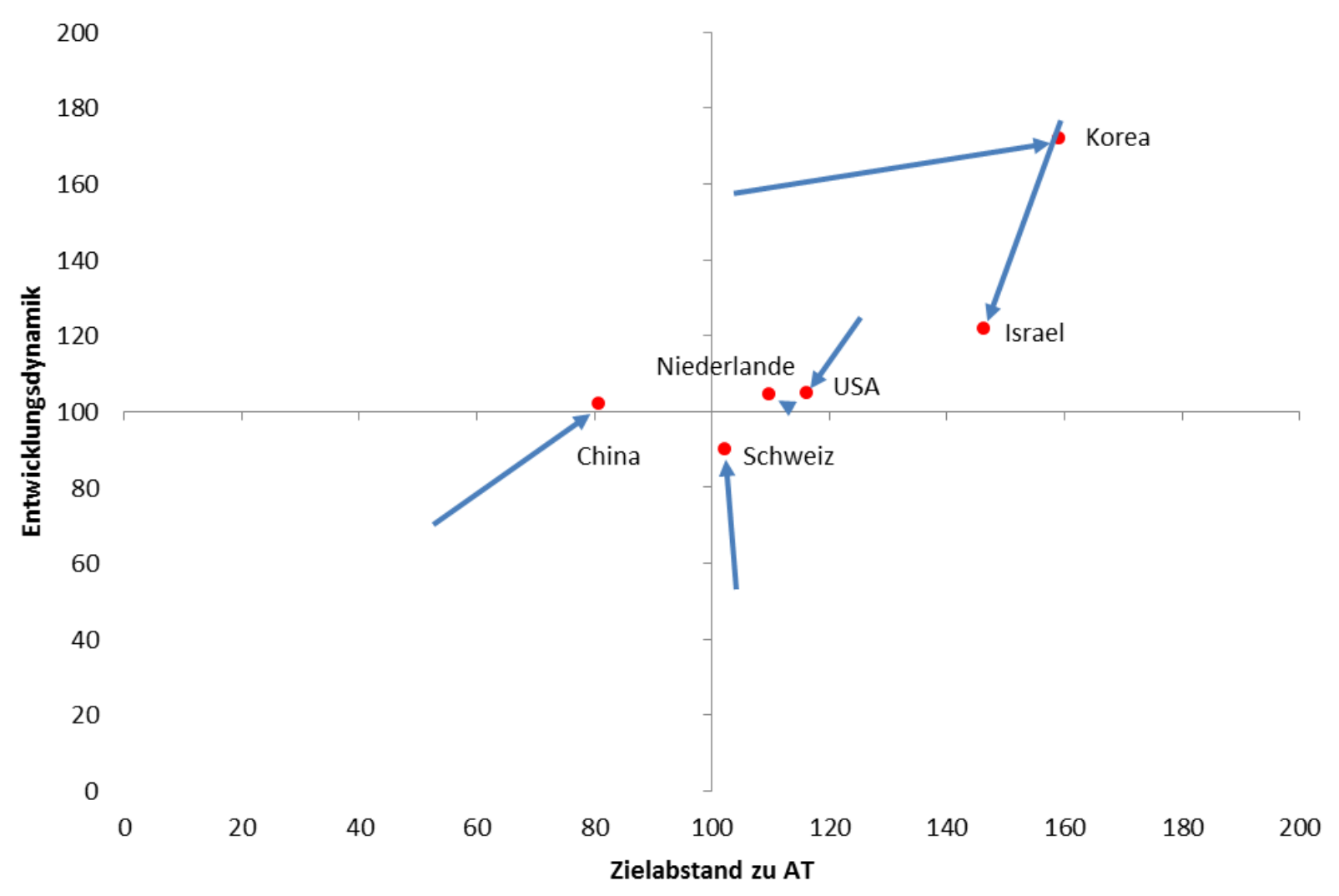

Quelle: siehe Indikatorenliste Anhang 1, WIFO-Darstellung; Erläuterungen zu Methodik und Interpretation der Abbildungen und Indikatoren siehe Anhang 2 


\section{FORSCHUNGSFINANZIERUNG}

Werden die großen Weltregionen hinsichtlich ihrer Entwicklung in Wissenschaft, Forschung und Innovation betrachtet, ist eine massive Umwälzung der Anteile betreffend der eingesetzten Finanzmittel zu beobachten.

In Relation zur Bevölkerung sind es neben den USA vor allem europäische Staaten, die hohe Ausgaben in F\&E sowohl pro Kopf als auch als Prozentsatz des BIP aufweisen (siehe Abbildung 6). Schweden, Finnland, Dänemark und die Schweiz sind hier zu nennen. Österreich liegt in dieser Statistik ebenfalls mit jeweils Platz 9 in den Jahren 2011 und 2012 in der Gruppe der forschungsintensivsten Länder der Welt. ${ }^{15}$ Im gleichen Ausmaß werden F\&E etwa auch in Singapur, Südkorea, Israel, Deutschland oder Japan gefördert. In dieser Statistik nimmt China nur einen hinteren Rang ein. Gemessen am Gesamtvolumen von mehr als 208 Mrd. US\$ liegt China jedoch bereits auf Platz 2 - hinter den USA (415 Mrd. US\$) und vor Japan (141 Mrd. US\$). ${ }^{16}$

Im Vergleich, mit etwa 10 Mrd. US\$ (kaufkraftbereinigt) an F\&E-Ausgaben im Jahr 2012 nimmt Österreich - was die absoluten F\&E-Ausgaben betrifft - in der globalen Liste der Länder den beachtlichen 20. Platz ein. Relativ zum BIP entspricht dies einer F\&E-Quote von ca. 2,8\% (siehe Abbildung 6). Österreich liegt damit derzeit auf dem 12. Rang. ${ }^{17}$

\section{Abbildung 6: F\&E-Quoten ausgewählte Länder}

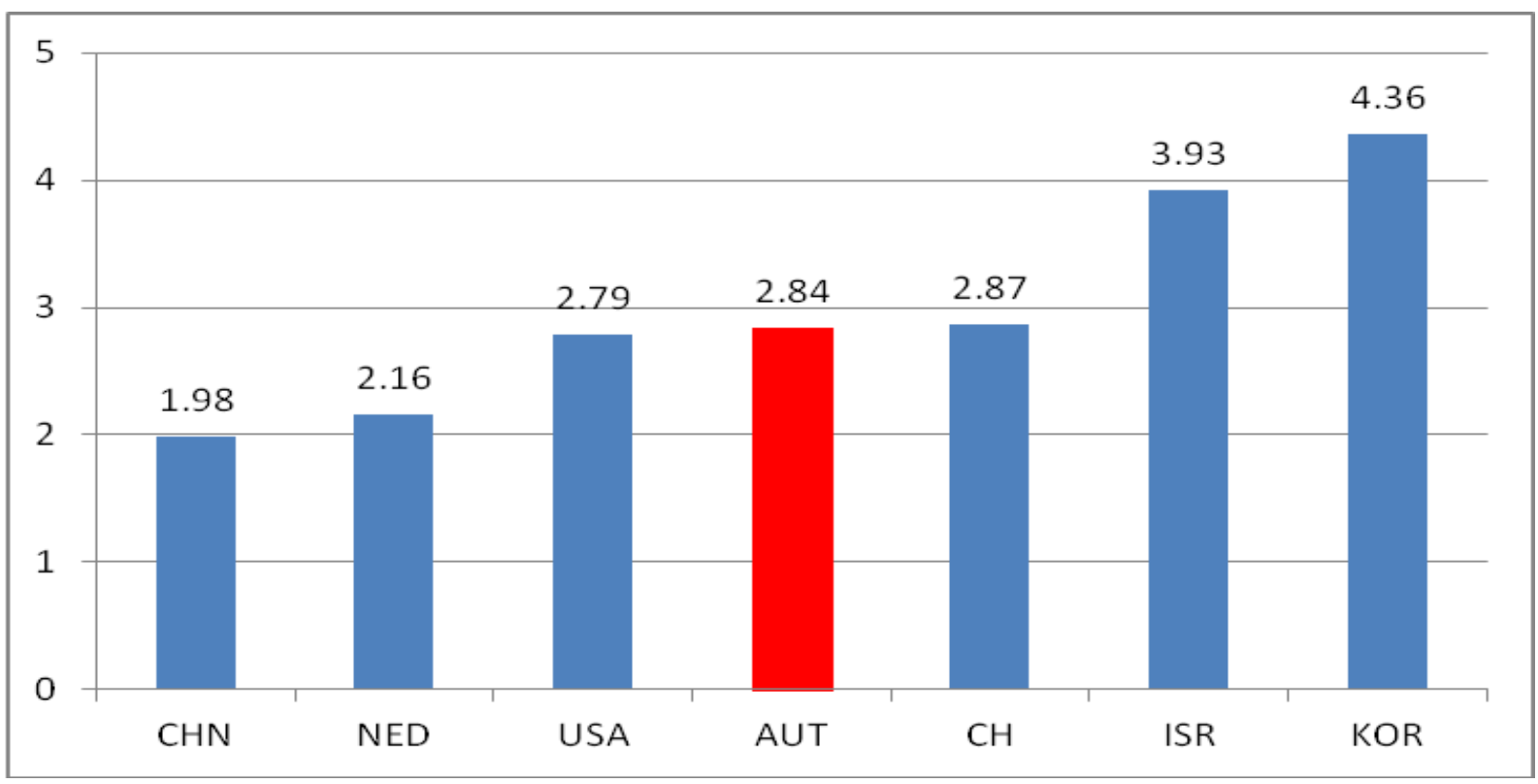

Quelle: OECD

\footnotetext{
15 OECD (2014): Main Science and Technology Indicators; GERD per capita population in 2011 and 2012.

16 OECD (2014): Gross domestic expenditure on R\&D, total (business enterprise, government, higher education, privat-non profit; data 2011 or most recent available).

${ }^{17}$ Vgl. IHS (2013): Vision Österreich 2050: Vorsprung durch Bildung, Innovation und Wandel, S. 74.
} 
Aufgrund der für Länder im Aufholprozess („catching-up“) typischen hohen Wachstumsraten befinden sich China ebenso wie Indien in einem rasanten Aufholprozess. So hat etwa China seine Aufwendungen von 2007 bis 2011 verdoppelt, zwischen 2003 und 2011 erhöhten sich die Aufwendungen sogar um das 4,5-fache.

2012 betrug Chinas F\&E-Quote bereits 1,98\% des BIP. Damit konnte China das erste Mal zur Europäischen Union aufschließen, deren Quote im selben Jahr $1,97 \%$ des BIP betrug. ${ }^{18}$ Das enorme Wachstum in China ist durch einen hohen Anteil an Forschungsausgaben von Unternehmen geprägt. Der Anteil der Unternehmen an der gesamten F\&E-Finanzierung betrug im Jahr 2012 74\%. Ein ähnlich hoher Anteil wird in Asien nur in Korea (73,7 \%) und Japan (76,5 \%) erreicht. Nennenswerte F\&EInvestitionen im asiatischen Raum sind allerdings auf die genannten Staaten plus Singapur und Malaysia beschränkt. Die Forschungsausgaben der weiteren Länder dieser Region bewegen sich soweit die Daten verfügbar sind - weit unter $0,5 \%$ des BIP für F\&E.

Die Investitionen in F\&E in den Ländern Mittel- und Südamerikas sind gering und auch die F\&EWachstumsraten erreichen nicht annähernd jene von asiatischen Vergleichsländern. Brasilien, mit einem F\&E-Anteil am BIP von $1,16 \%{ }^{19}$, und hier wiederum der Großraum São Paulo, sind hier mit einer nennenswerten Forschung und vergleichbaren tertiären Bildungseinrichtungen anzuführen. Für die Länder Argentinien, Chile, Mexiko oder Costa Rica liegt das F\&E-Aufkommen bei ca. 0,5\% des BIP. $^{20}$

Der Rückstand gegenüber beispielsweise dem ostasiatischen Raum kann zum Teil durch die schwache Bildungspolitik erklärt werden. Diese wird auch durch die unterdurchschnittlichen Leistungen im Rahmen der PISA-Studien sichtbar. Alle in dieser Statistik erfassten Staaten Lateinamerikas finden sich im unteren Drittel wieder. Die Prognosen für das Wirtschaftswachstum dieser Länder fallen aus diesem Grund auch langfristig nicht günstig aus.

Viel Unterstützung wird nötig sein, um die Länder des afrikanischen Kontinents - und das nicht nur im Bereich von Forschung und Innovation - an andere Regionen heranzuführen. ${ }^{21}$ Forschung in einem nennenswerten Ausmaß (über 0,5\% F\&E-Quote) findet laut den verfügbaren Statistiken nur in Tunesien, Marokko, Südafrika, Botswana und Gabun statt. ${ }^{22}$

\footnotetext{
18 OECD (2013): Main Science and Technology Indicators.

${ }^{19} \mathrm{http}: / /$ data.uis.unesco.org/index.aspx?queryid=74

${ }^{20}$ http://data.uis.unesco.org/index.aspx?queryid=74

${ }^{21}$ Das BIP des afrikanischen Kontinents entspricht mit rund 3200 Mrd. US \$ etwa jenem Deutschlands.

22 UNESCO (2012): Global investments in R\&D.
} 
Ziel des "Global Innovation Monitors" ist es, Österreich anhand ausgewählter Indikatoren, die auch im „Bericht zur wissenschaftlichen und technologischen Leistungsfähigkeit Österreichs“ verwendet werden, mit den führenden Innovationsnationen nicht nur auf europäischer Ebene, sondern im globalen Kontext zu vergleichen. Dazu wurden die Länder China, Israel, Niederlande, die Schweiz, Südkorea und die USA ausgewählt. Diese sind Zielländer der Prioritäten 1 und 2 aus den Empfehlungen der AG $7 \mathrm{a}$ an die Task Force $\mathrm{FTI}^{23}$ und gelten als globale Innovation Leaders, die aufgrund ihrer erfolgreichen Performance als Benchmark herangezogen werden. In der Folge werden für die erwähnten Länder jeweils kurze Profile präsentiert, die die Performance wesentlicher Bereiche des Innovationssystems sowie die allgemeine wirtschaftliche und soziale Leistungsfähigkeit in Form von zusammengesetzten Indikatoren in Relation zu Österreich abbilden.

\section{CHINA}

China hat sich in den letzten Jahren nicht nur zu einer globalen Wirtschaftsmacht, sondern zunehmend auch zu einem starken Player in der internationalen Forschungscommunity entwickelt. Parallel zu seinem Wirtschaftswachstum erhöht China auch seine F\&E-Ausgaben kontinuierlich: Die Forschungsquote liegt bereits heute bei $2 \%$ des BIP und der Trend geht steil nach oben. Auch die politischen Zielsetzungen sind ambitioniert: Ziel ist es, bis 2020 2,5\% des BIP zu erreichen. Bereits heute absolvieren rund 4 Millionen Studierende pro Jahr die staatlichen Universitäten. Die Anzahl an wissenschaftlichen Publikationen hat sich in der letzten Dekade verdreifacht. Seit 2011 führt China die internationale Patentstatistik mit über 500.000 jährlichen Patentanmeldungen an.

Abbildung 6 zeigt die Position Chinas relativ zu Österreich für ausgewählte Indikatoren. Nicht überraschend schneidet hier Österreich im Vergleich mit China als ein im Aufholprozess befindliches Land in beinahe allen Bereichen besser ab. Auch die Entwicklungsdynamik, anhand der die Zielerreichungschance gemessen werden kann, reicht nicht aus, um Österreich bis ins Jahr 2020 einzuholen. Dieses Bild könnte sich allerdings in den nächsten Jahren und Jahrzehnten ändern, sofern Österreich nicht genug und China weiterhin hohe Beträge in Zukunftsbereiche investiert.

Chinas erklärtes Ziel ist es, eine Wirtschaft aufzubauen, die zunehmend auf eigene Innovationen und weniger auf Imitationen gründet. Dafür wird die weitere Globalisierung des Innovationssystems vorangetrieben, wobei vor allem der Ausbau der internationalen Kooperationen von großer strategischer Relevanz ist. Ausländische High-Tech Unternehmen haben bis dato 3.300 F\&EEinrichtungen etabliert und damit rund 300.000 Forschungsarbeitsplätze geschaffen. Mehr als 300 der Fortune-500-Unternehmen haben Forschungszentren in China eingerichtet.

23 Beyond Europe: Die Internationalisierung Österreichs in Forschung, Technologie und Innovation über Europa hinaus. Empfehlungen der AG 7a an die Task Force der Bundesregierung (Juli 2013), S. 7. 


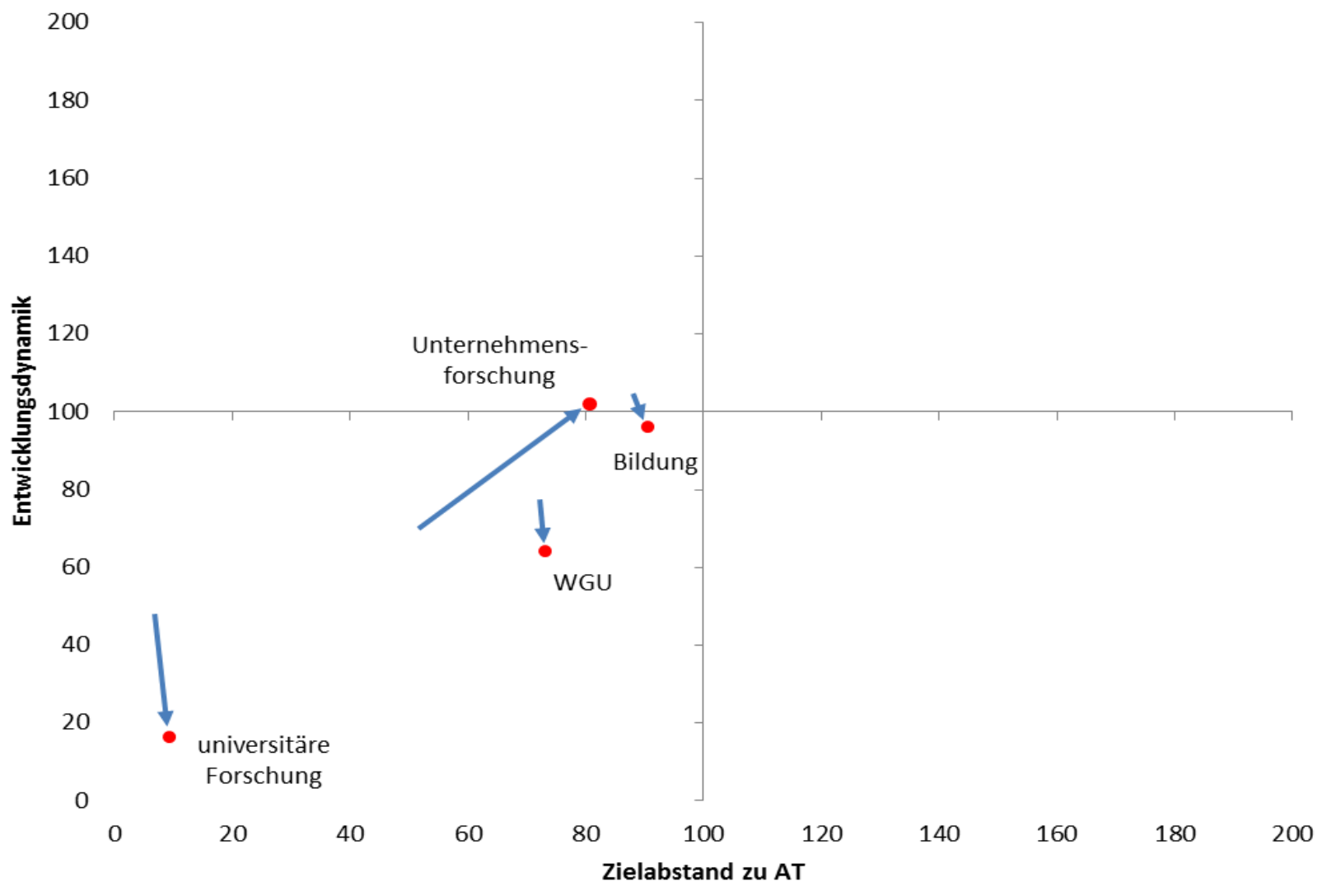

Quelle: siehe Indikatorenliste Anhang 1, WIFO-Darstellung; Erläuterungen zu Methodik und Interpretation der Abbildungen und Indikatoren siehe Anhang 2

Die Zahl der ForscherInnen in China steigt kontinuierlich. Im Jahr 2012 waren rund 3 Millionen ForscherInnen im Innovationssystem beschäftigt. Bis 2015 soll die Anzahl der Forscherlnnen pro 10.000 Beschäftigte auf 43 steigen. Mindestens 5\% der Bevölkerung sollen dann zumindest über eine wissenschaftliche Basisausbildung verfügen.

Der Output an Publikationen hat sich seit 2003 von 50.000 auf 165.000 mehr als verdreifacht. Im Bereich der Natur- und Ingenieurwissenschaften haben chinesische ForscherInnen ihren KollegInnen in den USA bei der Anzahl der Veröffentlichungen mittlerweile bereits den Rang abgelaufen. Wenn sich diese Trends weiter fortsetzen, wird China die USA bis 2015 auch bei der Gesamtzahl der Publikationen überholen. Allerdings offenbart diese Entwicklung auch Schwächen: Laut einer aktuellen Untersuchung von "Science" sind Manipulationen sowohl bei der Urheberschaft als auch bei den Forschungsarbeiten selbst weit verbreitet. Das Fazit von "Science“ bringt die Situation folgendermaßen auf den Punkt: In China geht es bei wissenschaftlichen Publikationen „wie auf einem Basar" zu. ${ }^{24}$

${ }^{24}$ Hvistendahl (2013): China's Publication Bazaar. In: Science, Vol. 342, no. 6162, pp. 1035-1039. 
ISRAEL

Israel zählt zu den innovativsten Ländern der Welt. Mit einer F\&E-Quote von über $4 \%$ nimmt es weltweit einen Spitzenplatz ein. Dafür ist vor allem der innovative und dynamische private Sektor verantwortlich, der rund $80 \%$ der F\&E-Ausgaben finanziert. Mit gut ausgebildetem Humankapital und exzellenten Forschungsleistungen liefern einige der weltweit führenden wissenschaftlichen Forschungseinrichtungen wie das Weizmann Institute of Science oder die Tel Aviv University die Grundlagen für eine zunehmend wissensbasierte Wirtschaft. Der medium- und high-tech-Sektor ist mittlerweile für annähernd die Hälfte der Exporte verantwortlich.

Dabei basierte der Großteil der Wirtschaftsleistung bis in die 1960er Jahre vor allem auf landwirtschaftlicher Produktion, und hier wiederum primär Orangen, deren Anteil am Export bis zu $60 \%$ des BIP betrug. Durch staatliche Initiativen wie das Inkubator-Programm und einem innovativen Unternehmenssektor ist es gelungen, einen großflächigen Strukturwandel herbeizuführen. Die Zahl der UnternehmensforscherInnen pro 1000 Beschäftigte ist mit rund 18 ForscherInnen annähernd vier Mal so hoch wie im EU-Durchschnitt $(4,6)$. Der Unternehmensbereich ist auch für eine überdurchschnittlich hohe Patentaktivität verantwortlich: Mit 2,5 triadischen Patentanmeldungen pro ForscherIn führt Israel vor Japan und Deutschland die internationale Patentstatistik an.

\section{Abbildung 8: Entwicklung ausgewählter Indikatoren Israels relativ zu Österreich}

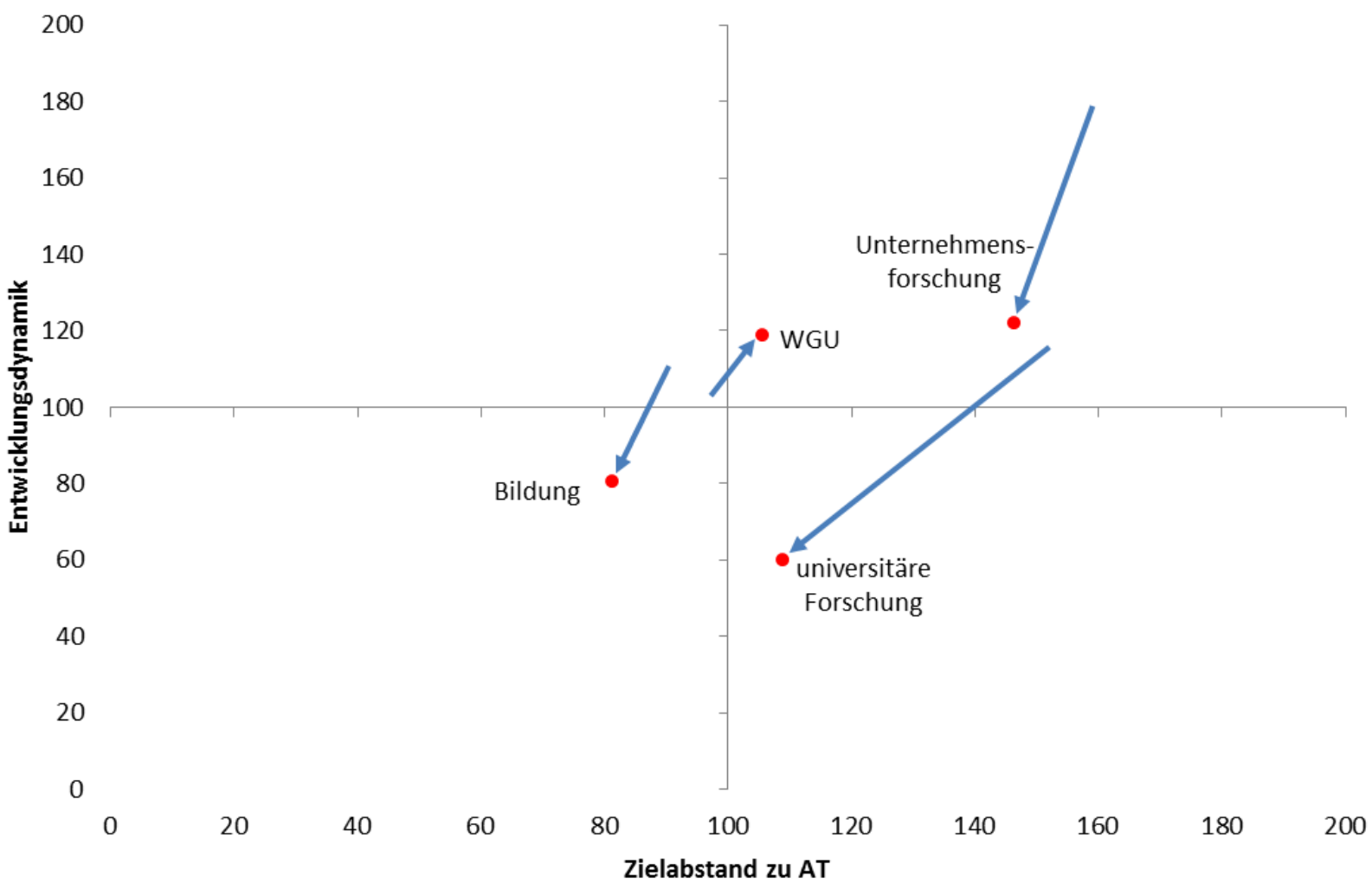

Quelle: siehe Indikatorenliste Anhang 1, WIFO-Darstellung; Erläuterungen zu Methodik und Interpretation der Abbildungen und Indikatoren siehe Anhang 2 
Abbildung 8 zeigt die Position Israels relativ zu Österreich für ausgewählte Indikatoren. Hier bestätigt sich die Position Israels als Innovation Leader: bis auf das Bildungssystem besteht bei allen Indikatoren ein positiver Abstand zu Österreich.

Die herausragende Besonderheit Israels ist die hohe Gründungsaktivität: Pro Kopf hat Israel heute mehr high-tech Start-ups und eine höhere Risikokapitalintensität als jedes andere Land der Welt. Die Stadt Tel Aviv sowie ihre Umland und das sogenannte Silicon Wadi gelten nach dem Silicon Valley als zweitattraktivste Technologie- und Start-up-Region der Welt. Entsprechend sind mit über 60 Firmen mehr israelische Unternehmen an der Technologiebörse NASDAQ gelistet als aus irgendeinem anderen Land. Das Wirtschaftswachstum lag daher in den Jahren vor der globalen Finanz- und Wirtschaftskrise konstant bei über $5 \%$ und bewegt sich seither zwischen 3 und $4 \%$. 


\section{KOREA, REPUBLIK}

Südkorea hat einen bemerkenswerten Aufstieg hinter sich. Noch in den 1960er Jahren gehörte es zu den ärmsten Ländern der Welt. Heute ist die Republik Korea die viertgrößte Volkswirtschaft in Asien und als Mitglied der G-20 Staaten eine der leistungsfähigsten und größten Volkswirtschaften weltweit. ${ }^{25}$ Die Ausgaben für Forschung und Entwicklung als Prozentsatz des BIP haben sich in den letzten zehn Jahren beinahe verdoppelt, mit einem Wert von 4,4\% belegte Korea im Jahr 2012 einen internationalen Spitzenrang. ${ }^{26}$ Bis 2018 soll die Forschungsquote auf $5 \%$ angehoben werden.

\section{Abbildung 9: Entwicklung ausgewählter Indikatoren Koreas relativ zu Österreich}

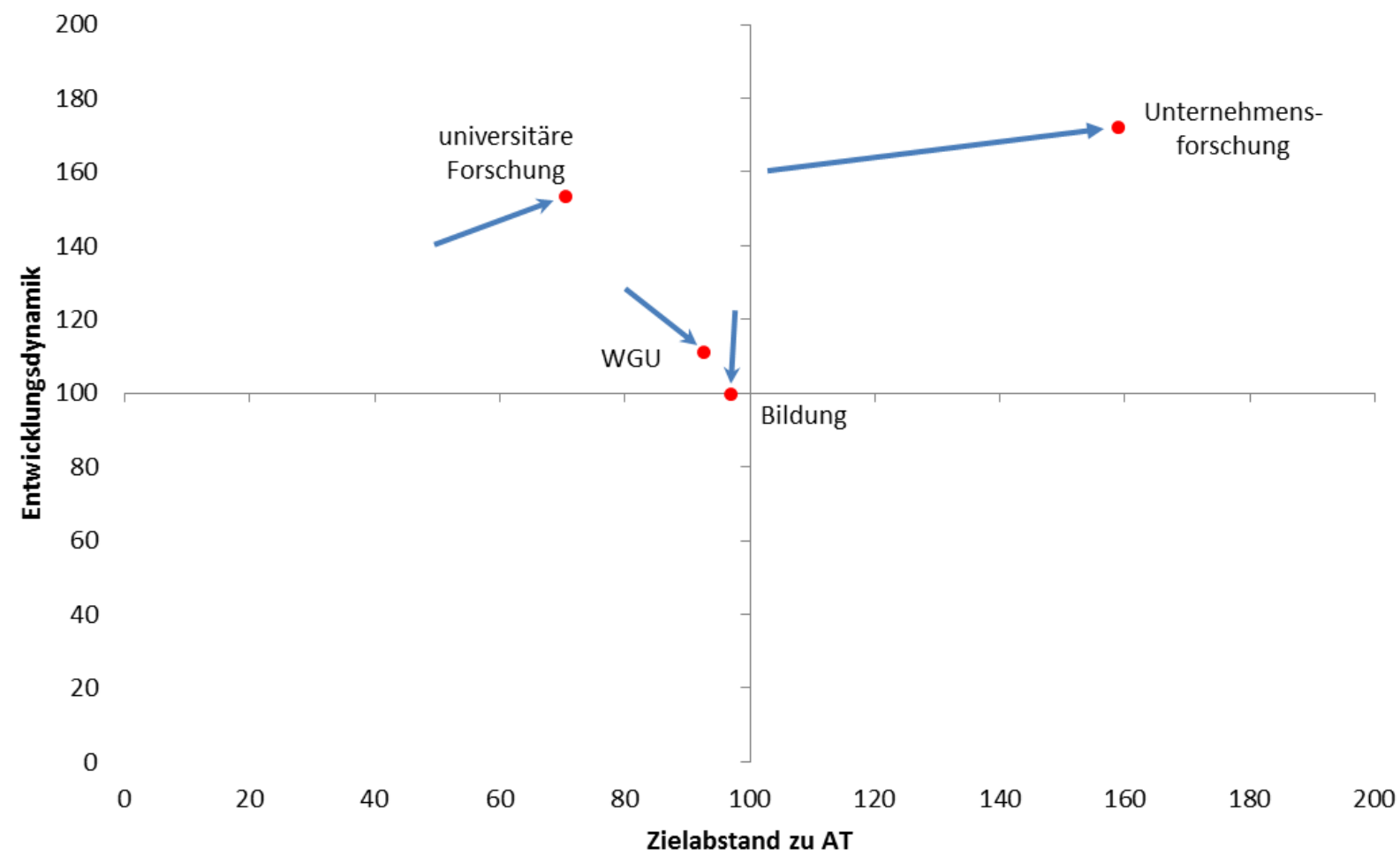

Quelle: siehe Indikatorenliste Anhang 1, WIFO-Darstellung; Erläuterungen zu Methodik und Interpretation der Abbildungen und Indikatoren siehe Anhang 2

Abbildung 9 zeigt die Position Koreas relativ zu Österreich für ausgewählte Indikatoren. Hier zeichnet sich ein für Korea sehr positives Bild ab: entweder weisen die Indikatoren zwar einen negativen Abstand zu Österreich auf, aber eine hohe Dynamik in Form einer Zielerreichungschance von größer

\footnotetext{
${ }^{25}$ Wird das BIP als Maßstab genommen, so liegt Südkorea auf Rang 12 der weltweiten Volkswirtschaften; vgl. IMF (2014): World Economic Outlook Database.

${ }^{26}$ Vgl. OECD (2014): Main Science and Technology Indicators.
} 
als 100 (d.h. Österreich wird bis 2020 eingeholt werden), oder sie bilden bereits einen positiven Abstand zu Österreich ab. All dies ist Resultat eines massiven Investitionsprozesses in unterschiedlichen Bereichen (Bildungssystem, Unternehmenssystem). Diese Entwicklung weist auch darauf hin, dass Österreich gegenüber im Aufholprozess befindlichen Ländern bereits relativ kurzfristig ins Hintertreffen geraten könnte.

Ablesen lässt sich das etwa an den überdurchschnittlich hohen privaten F\&E-Investitionen, die rund 75\% der gesamten Forschungsausgaben Koreas ausmachen und hier wiederum zu einem großen Anteil auf Großunternehmen konzentriert sind. Die vier größten Unternehmen (Samsung, LG, Hyundai, SK) sind für annähernd $60 \%$ der Forschungsausgaben verantwortlich. Der Fokus der Forschung liegt dabei auf der IT-Branche. Korea versucht seine starke Abhängigkeit einerseits von großen Unternehmen andererseits von einem dominierenden Wirtschaftssektor nun spezifisch durch verstärkte Förderung von KMU abzufedern. Besonders in Kombination mit der "creative economy" werden Unternehmensgründungen und Start-ups unterstützt.

Auf Bildung wird in Asien traditionell sehr hoher Wert gelegt. In Korea ist das nicht anders: Regelmäßig belegen koreanische SchülerInnen einen der vorderen Plätze in den PISA-Rankings. Der Druck, der dabei auf SchülerInnen und StudentInnen lastet, ist allerdings groß. Eine der Folgen ist die zum Teil beträchtliche Belastung der Familieneinkommen durch private Ausgaben für Bildung. Laut einer OECD Studie ${ }^{27}$ liegt der private Finanzierungsanteil für tertiäre Bildung in Korea bei etwa $74 \%$. Zum Vergleich: in Österreich liegt dieser bei 12,3\%.

Rund $80 \%$ der AbsolventInnen der Sekundarstufe II (Oberstufe) streben ein Studium an einer der rund 400 öffentlichen und privaten Universitäten an. Bei den besten Universitäten des Landes herrscht daher ein selektiver Aufnahmeprozess. Korea versucht in den letzten Jahren auch verstärkt international WissenschafterInnen zu akquirieren, insbesondere um das Leistungsniveau zu heben und koreanische Topuniversitäten noch stärker an die Spitze zu führen.

27 OECD (2013): Private Ausgaben für die tertiäre Bildung. In OECD (2013): Die OECD in Zahlen und Fakten: Wirtschaft, Umwelt, Gesellschaft. 


\section{NIEDERLANDE}

Die Niederlande gehören laut dem Global Competitiveness Report 2013-2014 zu den zehn wettbewerbsfähigsten und innovativsten Nationen weltweit, und dies trotz des Umstandes, dass sie im Hinblick auf die Investitionen in Forschung und Entwicklung mit einer F\&E-Quote von 2,16\% des BIP (2012) nur einen mittleren Rang in der OECD belegen. Besonders herausgestrichen wird im Global Competitiveness Report das sehr gute niederländische Bildungswesen (Platz 4 bei „health and primary education“ und Platz 6 bei "higher education and training") und die exzellente Infrastruktur. ${ }^{28}$

Abbildung 10 zeigt die Position der Niederlande relativ zu Österreich für ausgewählte Indikatoren.

\section{Abbildung 10: Entwicklung ausgewählter Indikatoren der Niederlande relativ zu Österreich}

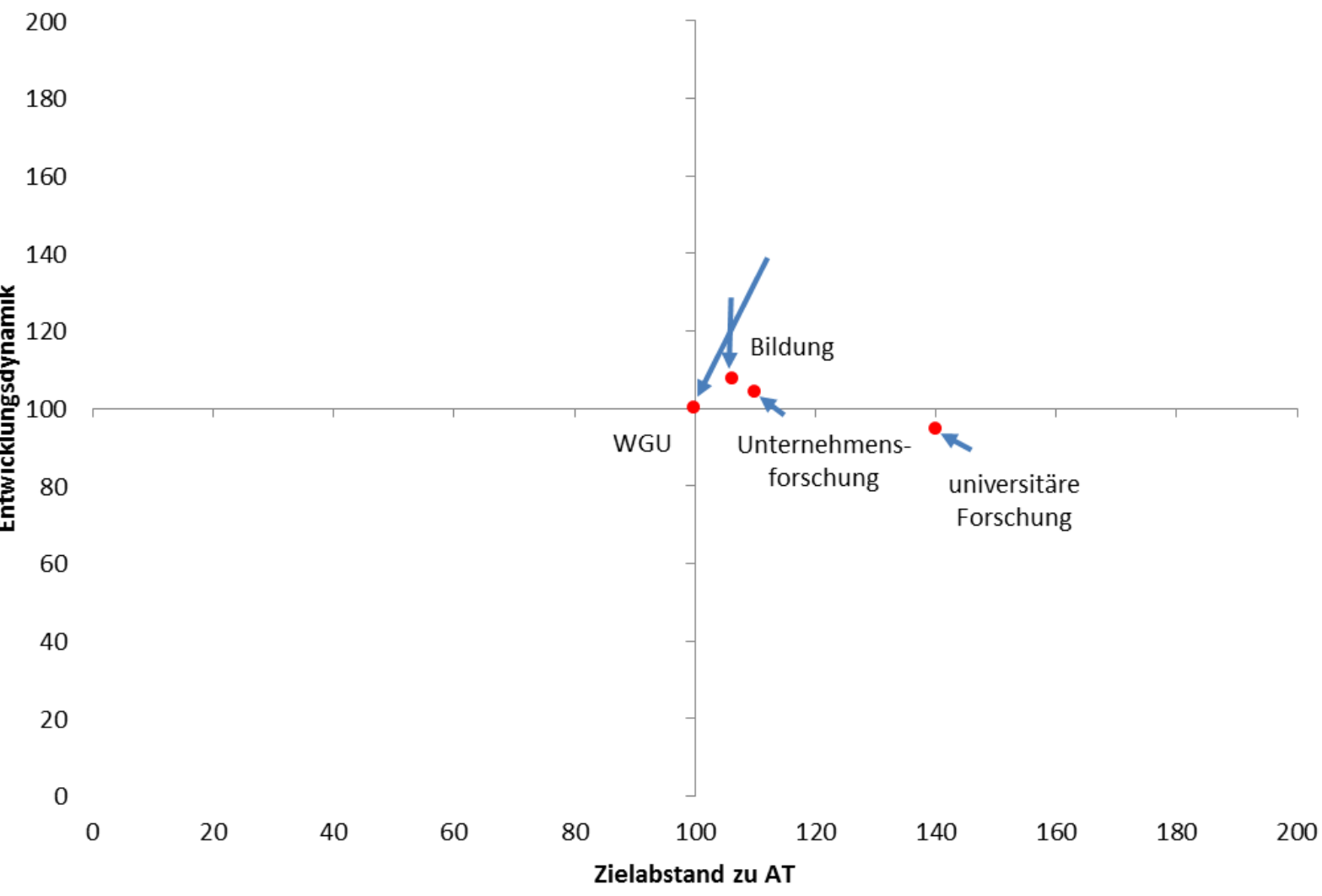

Quelle: siehe Indikatorenliste Anhang 1, WIFO-Darstellung; Erläuterungen zu Methodik und Interpretation der Abbildungen und Indikatoren siehe Anhang 2

\footnotetext{
28 World Economic Forum (2013): Global Competitiveness Report 2013-2014, S. 24.
} 
Hier schneiden die Niederlande in Summe geringfügig besser ab. Die Unterschiede sind allerdings nicht markant, so dass bei unveränderter Entwicklungsdynamik in den nächsten Jahren keine gravierenden Veränderungen zu erwarten sind.

Besonders die niederländischen Universitäten sind im internationalen Vergleich sehr erfolgreich. So erreichten im „Times Higher Education Supplement Ranking 2013-14“ insgesamt acht Universitäten aus den Niederlanden einen Platz unter den ersten 100 im weltweiten Vergleich, bzw. 12 einen Platz unter den ersten 200. Österreich konnte nur mit der Universität Wien einen Platz unter den ersten 200 erreichen. ${ }^{29}$

Das niederländische Wissenschaftssystem ist hochgradig vernetzt. Aufgrund der relativ schmalen wissenschaftlichen Basis (bedingt durch die niedrige Bevölkerungszahl) ist diese Vernetzung eine wichtige Bedingung für den Erfolg niederländischer Forschung.

Der Anteil öffentlich finanzierter F\&E ist relativ groß. Die Gründe dafür liegen einerseits in der stark auf Dienstleistungen ausgerichteten niederländischen Wirtschaft, andererseits in der Konzentration von F\&E in einigen wenigen großen Unternehmen (z.B. Philips). Um künftig mehr KMU am Innovationsprozess $\mathrm{zu}$ beteiligen, hat die niederländische Regierung vor einigen Jahren das Instrument der „Innovationsgutscheine“ eingeführt.

${ }^{29}$ http://www.timeshighereducation.co.uk/world-university-rankings/2013-14/world-ranking 


\section{SCHWEIZ}

Die Schweiz hat es in den letzten Jahren durch gemeinsame Anstrengungen aller Verantwortlichen geschafft, sich zu einem Innovationsland der Spitzenklasse zu entwickeln. Hierzu hat die schweizerische Wirtschaft, die sich von der weltweiten Konjunkturabkühlung nach 2008 überraschend schnell und kräftig erholen konnte, ebenso ihren Teil beigetragen wie die Politik mit ihren Weichenstellungen in den Bereichen Bildung, Wissenschaft und Forschung, Gesundheit und Pensionen. So verwundert es nicht, dass die Schweiz auch in fast allen Rankings zu Innovationsfähigkeit, Wettbewerbsfähigkeit, Lebensstandard, sozialer Situation oder Einkommensverteilung sehr gut abschneidet.

So konnte sich die Schweiz etwa im aktuellen "World Competitiveness Report 2014" des renommierten Lausanner Instituts für Management-Entwicklung (IMD) erneut auf Platz 2 behaupten, nur knapp geschlagen von den USA, die ihre Spitzenposition verteidigten. Im „Innovation Union Scoreboard" wird die Schweiz seit mehreren Jahren als innovativstes Land ausgewiesen. Lediglich in vier der 25 Indikatoren liegt die Schweiz nicht über dem Durchschnitt der EU-28. Vor allem in ihrem Wachstum, ihrer Innovationsdynamik und in ihrem wissenschaftlichen Output übertrifft sie jedoch die Mitgliedstaaten der EU bei weitem.

\section{Abbildung 11: Entwicklung ausgewählter Indikatoren der Schweiz relativ zu Österreich}

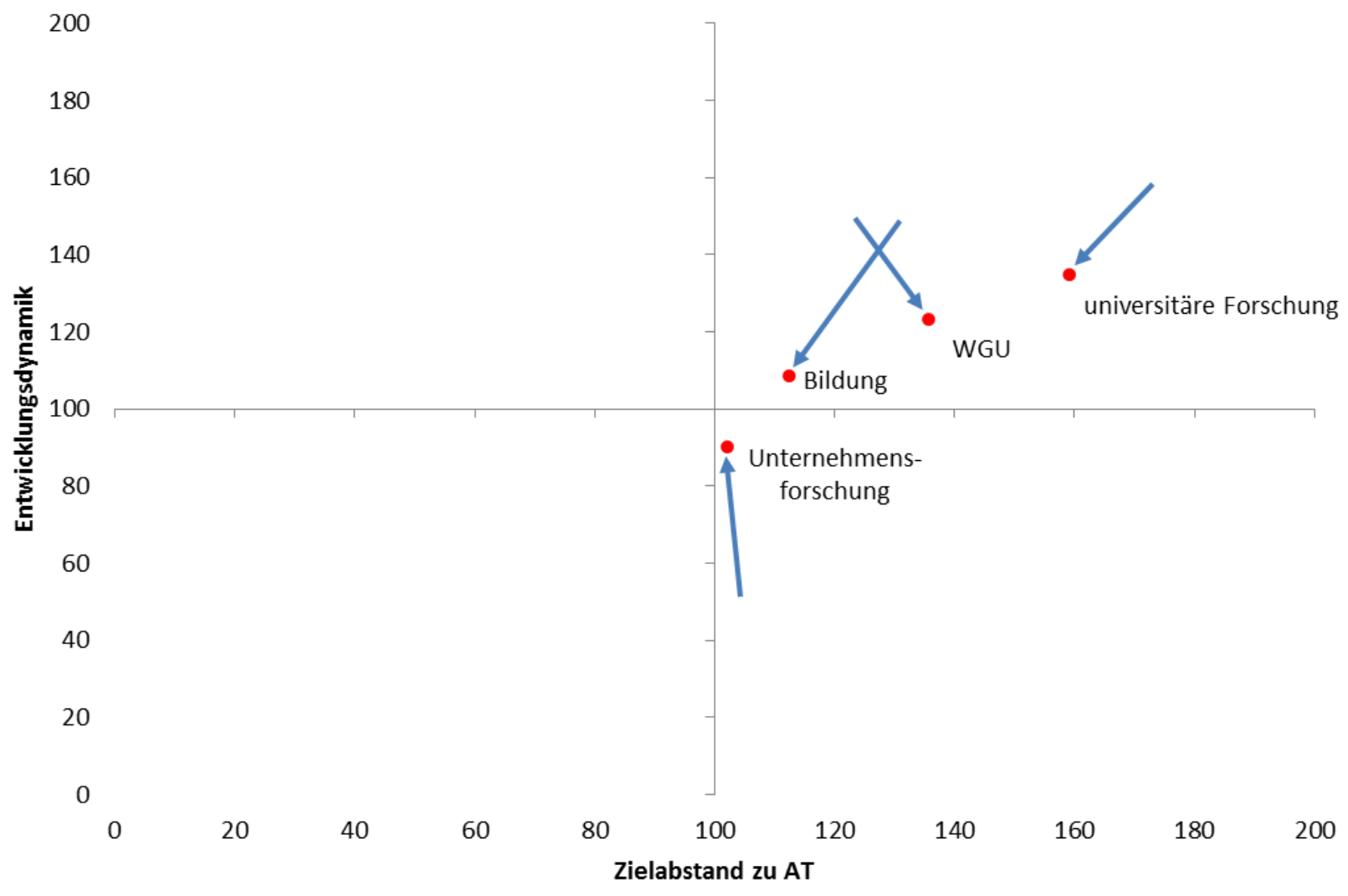

Quelle: siehe Indikatorenliste Anhang 1, WIFO-Darstellung; Erläuterungen zu Methodik und Interpretation der Abbildungen und Indikatoren siehe Anhang 2 
Abbildung 11 zeigt die Position der Schweiz relativ zu Österreich für ausgewählte Indikatoren. Nicht überraschend schneidet die Schweiz als innovationsführendes Land hier in allen Bereichen (in Form eines positiven Zielabstandes zu Österreich) besser ab, einzig bei der Unternehmensperformance liegt die Zielerreichungschance der Schweiz unter 100, zeigt jedoch eine verbesserte Dynamik seit 2001. Insgesamt spiegelt dieses Bild die enormen finanziellen Anstrengungen der Schweiz in den entsprechenden Bereichen in den letzten Jahren wider.

Die überdurchschnittliche wissenschaftliche Performance der Schweiz hängt zum Teil mit der langen Tradition eines sehr autonom agierenden, international ausgerichteten Wissenschaftssystems zusammen. Eine Folge daraus ist sicher, dass etwa die Eidgenössisch Technische Hochschule (ETH) Zürich in den diversen Universitätsrankings zum wiederholten Mal unter die Top 15 Universitäten weltweit und zur besten Hochschule im deutschen Sprachraum gereiht wurde. Ebenso gibt es offenbar einen gesellschaftlichen Konsens über die Bedeutung von Wissenschaft und Forschung für die Gesellschaft und die Wirtschaft, was zu vergleichsweise hohen privaten und öffentlichen Investitionen in diesen Bereich geführt hat. Außerdem verfügt die Schweiz über eine ausgezeichnete Innovationsbasis in ihrer Unternehmensstruktur: Neben hoch innovativen, großen, multinational agierenden "Global Players" mit Schweizer Ursprung existiert eine Vielzahl von innovativen KMU. Auch der Dienstleistungssektor ist sehr innovationsorientiert. Hier spielen vor allem Finanzdienstleistungen eine bedeutende Rolle. 


\section{USA}

Abbildung 12 zeigt die Position der USA relativ zu Österreich für ausgewählte Indikatoren. Das sich hier abzeichnende Bild ist nicht ganz eindeutig: Einerseits ist - nicht überraschend - die Unternehmensperformance der USA besser als jene Österreichs, andererseits schneidet, wie bereits eingangs erwähnt, die USA bei der Performance von Wirtschaft, Gesellschaft und Umwelt, die letztendlich das „well-being“ eines Landes reflektieren, schlechter ab. Die Dynamik bei der universitären Forschung ist im Vergleich zu 2001 etwas zurückgegangen, es besteht aber immer noch eine positive Lücke zu Österreich. Kaum Unterschiede (was die indikatorenbasierte Evaluierung betrifft) gibt es in Bezug auf das Bildungssystem.

\section{Abbildung 12: Entwicklung ausgewählter Indikatoren der USA relativ zu Österreich}

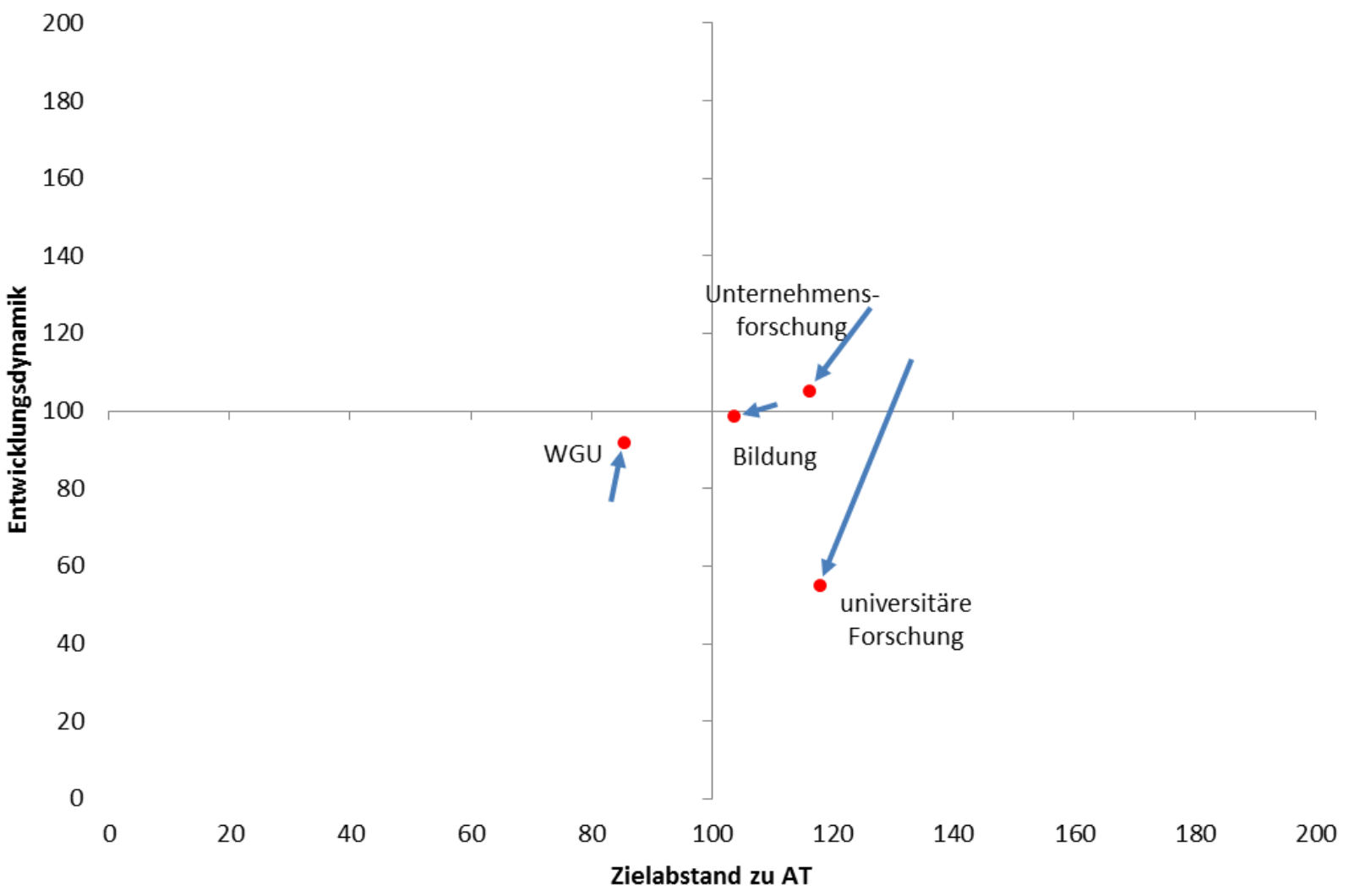

Quelle: siehe Indikatorenliste Anhang 1, WIFO-Darstellung; Erläuterungen zu Methodik und Interpretation der Abbildungen und Indikatoren siehe Anhang 2

Rund ein Drittel der weltweiten F\&E-Ausgaben im Jahr 2011 entfallen auf die USA. Für 2014 werden 465 Milliarden US\$ genannt, was einem Anteil von 2,8\% am BIP entspricht. ${ }^{30}$ In den letzten Dekaden

30 Vgl OECD (2013): Main Science and Technology Indicators. 
gab es positive Entwicklungen, jedoch sind die USA im globalen Vergleich - insbesondere relativ zu den asiatischen Staaten - zurückgefallen. Der weltweite F\&E-Anteil der USA ist von 37\% (2001) auf knapp unter 30\% (2011) gesunken. Die globale Krise hat zwar einen deutlichen Rückgang bei den F\&E-Ausgaben verursacht, jedoch sind seit 2010 wieder starke Zuwächse - insbesondere im privatwirtschaftlichen Sektor - erkennbar.

Der Gesamtaufwand für F\&E betrug im Jahr 2011 rund US\$ $425 \mathrm{Mrd}$. Inflationsbereinigt entspricht das dem Niveau von 2008. Dabei entfallen rund 63\% auf den Privatsektor und $30 \%$ auf die öffentliche Hand. Innerhalb der thematischen Bereiche kommt es hinkünftig zu Umschichtungen der verfügbaren Mittel. So sind die Zuwendungen der Regierung bei gesundheitsrelevanter Forschung (insbesondere durch das National Institute of Health) von 12\% im Jahr 1980 auf 22\% im Jahr 2011 gestiegen. Parallel dazu gehen die Investitionen in Verteidigungs- und Weltraumforschung weiter zurück. Andere Bereiche wie Energieforschung, Life Science, IKT und Advanced Materials werden hingegen verstärkt gefördert.

Die tertiäre Bildung hat einen gesellschaftlich hohen Stellenwert. Eine genauere Analyse der AbsolventInnenzahlen zeigt starke Zuwächse bei den Bachelor-Abschlüssen. So stieg die Anzahl der Bachelor-AbsolventInnen von etwa $400.000 \mathrm{im}$ Jahr 2000 auf etwa $560.000 \mathrm{im}$ Jahr 2011. Die soziale Selektion ist nach wie vor hoch, verbessert sich aber zunehmend, was auf unterschiedliche Maßnahmen der Regierung zurückzuführen ist.

Die zunehmende Bedeutung der internationalen Vernetzung beeinflusst auch in den USA die Entwicklungen im Forschungsbereich. Neben der akademischen Vernetzung ist auch der Markt für Innovationen aus den USA stark globalisiert. Die wissens- und technologiebasierten Wirtschaftsbereiche dominieren die Industrie und haben mit etwa $40 \%$ den größten Anteil am BIP. 
Anhang 1: Indikatoren-Set

\begin{tabular}{|c|c|c|}
\hline & Indikator & Quelle \\
\hline \multirow{10}{*}{$\begin{array}{l}\text { Wirtschaft, } \\
\text { Gesellschaft, Umwelt }\end{array}$} & BIP pro Kopf zu Kaufkraftstandards (KKS) & Eurostat \\
\hline & Erwerbstätigenquote & Eurostat \\
\hline & Erwerbstätigenquote China & Weltbank \\
\hline & Arbeitslosenquote & Eurostat \\
\hline & Arbeitslosenquote & Weltbank \\
\hline & Lebenserwartung in Gesundheit: Anteil der Jahre ohne & Eurostat \\
\hline & Krankheit/Beeinträchtigung an Lebenserwartung & \\
\hline & Greenhouse gases/GDP & Eurostat \\
\hline & Effizienzsteigerung: Energieintensität & Eurostat; Statistik Austria \\
\hline & Non-energy material productivity, US\$ per kg & Eurostat; Statistik Austria \\
\hline \multirow[t]{5}{*}{ Bildung } & Expenditure per student, tertiary (\% of GDP per capita) & OECD \\
\hline & Expenditure per student, All tertiary education & OECD \\
\hline & PISA mean score reading & OECD \\
\hline & PISA mean score mathematics & OECD \\
\hline & PISA mean score science & OECD \\
\hline \multirow[t]{3}{*}{ GF, Unis } & Leiden-Ranking & Leiden-Ranking \\
\hline & HERD/population 2005 US\$ PPP & OECD \\
\hline & Basic research expenditure as a percentage of GDP & OECD \\
\hline \multirow[t]{7}{*}{ Unternehmen } & Industry-financed GERD as a percentage of GDP & OECD \\
\hline & GERD as a percentage of GDP & OECD \\
\hline & $\begin{array}{l}\text { Privater Forschungsfinanzierungsanteil (financed by } \\
\text { industry \& abroad) }\end{array}$ & OECD \\
\hline & Total researchers per thousand labour force & OECD \\
\hline & Patent applications per million GDP & OECD \\
\hline & Doing Business (Starting a business) & Weltbank \\
\hline & Percentage of GERD by Business Enterprise sector & OECD \\
\hline
\end{tabular}


Der Vergleich des österreichischen Innovationssystems mit den ausgewählten Ländern beruht auf den erhobenen Daten für die in Anhang 1 angeführten Indikatoren, wobei der letztverfügbare Wert Österreichs in den unterschiedlichen Bereichen als Zielabstand, herangezogen wird.

\section{Abbildung 13: Musterabbildung}

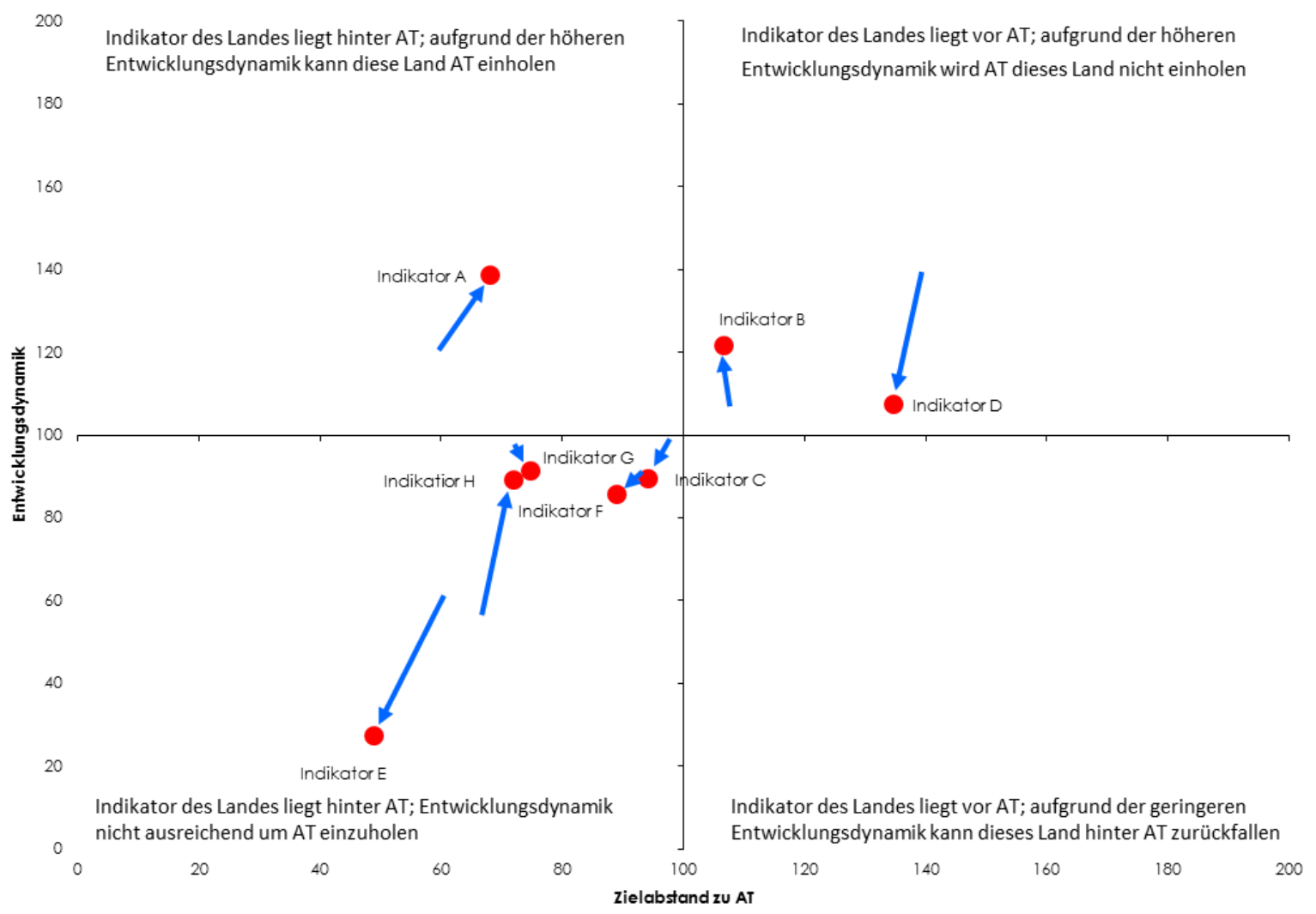

Die Indikatoren werden nach ihrem Abstand zu Österreich (Zielabstand zu AT) und ihrer Dynamik im Vergleich zu Österreich (Entwicklungsdynamik) dargestellt. Der Zielabstand reflektiert den Wert des jeweiligen Indikators eines Lands im Vergleich zum österreichischen Ist-Wert. Werte über 100 zeigen, dass sich das jeweilige Land über dem österreichischen Wert befindet. Der Zielabstand sagt damit etwas über den Niveauunterschied zu den Indikatoren Österreichs aus - über die derzeitige Performance eines Landes relativ zu Österreich, nicht aber über die langfristige Dynamik. So könnte sich ein Indikator, der derzeit nur knapp unter Ziel liegt, aufgrund einer negativen Dynamik weiter verschlechtern. Anders gesagt ermöglicht der ausschließliche Vergleich der Zielabstände keine Rückschlüsse auf die Wahrscheinlichkeit der zukünftigen Entwicklung.

Aus diesem Grund wurde als zweite Dimension der indikatorbasierten Darstellung die Entwicklungsdynamik auf der senkrechten Achse gewählt: Sie zeigt, ob das vergangene Wachstum des Indikators für die Erreichung des österreichischen Werts bis 2020 ausreichend ist. Sie ist das Verhältnis des Projektionswerts des jeweiligen Lands zum Wert für Österreich im Jahr 2020 (beruhend auf der durchschnittlichen jährlichen Wachstumsrate der Vergangenheit, d.h. unter einer 
„business as usual“ Annahme, d. h. alles läuft so weiter wie bisher). Werte über 100 zeigen, dass das Land bis 2020 Österreich einholen bzw. vor Österreich bleiben wird. Die Pfeile in den Abbildungen stellen den Verlauf der Entwicklungsdynamik seit 2001 dar. Der Endpunkt (= roter Punkt) ist der jeweilige Wert für 2012.

Neben dem zur Verfügung stehenden Zahlengerüst basieren die Beschreibungen und Einblicke in die Strukturen und Funktionsweisen anderer nationaler Innovationssysteme zum Teil auf existierenden Vergleichsstudien und Länderprofilen sowie auf Basis gesammelter Daten aus Delegationsreisen des Rates in leistungsfähige und forschungsstarke Länder. 